\title{
Sm-Nd isotope geochemistry and tectonic setting of the metasedimentary rocks from the basal allochthonous units of NW Iberia (Variscan suture, Galicia)
}

\author{
José Manuel Fuenlabrada ${ }^{a}$, Ricardo Arenas ${ }^{b, *}$, Rubén Díez Fernández ${ }^{\mathrm{c}}$, Sonia Sánchez Martínez ${ }^{\mathrm{b}}$, \\ Jacobo Abati ${ }^{\mathrm{b}}$, Alicia López Carmona ${ }^{\mathrm{b}}$ \\ a CAI de Geocronología y Geoquimica Isotópica, Facultad de Geología, Universidad Complutense de Madrid, 28040 Madrid, Spain \\ b Departamento de Petrologia y Geoquimic e Instituto de Geociencias (UCM-CSIC), Universidad Complutense de Madrid. 28040 Madrid, Spain \\ 'Departamento de Geología, Universidad de Salamanca, 37008 Salamanca, Spain
}

Keywords:

Metasedimentary rocks

Tectonic setting

Sm-Nd isotope geochemistry

Allochthonous complexes

Variscan belt

NW Iberia

\begin{abstract}
A B S T R A C T
The basal units of the allochthonous complexes of NW Iberia are formed by thick metasedimentary rock sequences intruded by granitoids, ranging in composition from calc-alkaline (c. $493 \mathrm{Ma}$ ) to minor alkalineperalkaline massifs (c. 475-470 Ma), and mafic rocks. The granitoids were transformed into variably deformed othogneisses and the associated mafic rocks were transformed into amphibolites, blueschists and eclogites during eo-Variscan high-P metamorphism dated at c. $370 \mathrm{Ma}$. Two different superimposed metasedimentary rock sequences can be distinguished. The lower sequence (maximum depositional age at c. $560 \mathrm{Ma}$ ) is mainly composed of metagreywackes, while the upper sequence (maximum depositional age at c. $500 \mathrm{Ma}$ ) consists of mica schists and other minor types. Major and trace element geochemistry of the metagreywackes of the lower sequence suggests that they were generated in relation to a peri-Gondwanan arc system built on the thinned continental margin, although some chemical transition to passive margin greywackes is also observed. This sedimentary sequence was probably deposited in an Ediacaran-Early Cambrian back-arc setting or retro-arc setting, closer to the thinned platform of the continental margin. The geochemical features of the sedimentary rocks of the upper sequence suggest some affinity with passive margin sediments; they were probably deposited closer to the continental domain and to certain distance from the most active zones of the magmatic arc. The Nd model ages of 23 analysed samples are Paleoproterozoic and range between $1782 \mathrm{Ma}$ and $2223 \mathrm{Ma}$ (average value $1919 \mathrm{Ma}$ ). The $\mathrm{Nd}$ model ages are slightly younger in the upper sequence than in the lower sequence, but altogether they define a single population, and therefore the two metasedimentary rock sequences can be clearly related. Sedimentation probably took place within the same basin located in the continental platform of Gondwana, the main source areas of these sedimentary rocks did not change during the late Neoproterozoic and Cambrian times. The Nd model ages are very old and they seem to be compatible with Paleoproterozoic or Archean source areas, with only minor participation of younger sources probably represented by intrusive Cadomian-Pan-African granitoids.
\end{abstract}

\section{Introduction}

The interpretation of metasedimentary series involved in the suture zone of mountain belts is a key issue to unravel the origin of the most relevant terranes, frequently with different tectonic settings and sometimes characterized by an important exoticism (Hatcher et al., 2007; Merschat and Hatcher, 2007; Searle, 2007). These are generally highly deformed and variably metamorphosed azoic metasedimentary series;

\footnotetext{
* Corresponding author at: Departamento de Petrología y Geoquímica e Instituto de Geociencias (UCM-CSIC). Facultad de Geología, Universidad Complutense de Madrid, C/ José Antonio Novais, no 2, 28040 Madrid, Spain. Tel.: + 34 639601919 ; fax: + 34915442535.

E-mail addresses: jmfuenla@pas.ucm.es (J.M. Fuenlabrada), arenas@geo.ucm.es (R. Arenas),georuben@usal.es (R. Díez Fernández), s.sanchez@geo.ucm.es (S. Sánchez Martínez), abati@geo.ucm.es (J. Abati), alcarmona@geo.ucm.es (A. López Carmona).
}

consequently little information can be obtained from traditional studies based in paleontology or stratigraphy. In these cases, provenance analysis based in $\mathrm{U}-\mathrm{Pb}$ dating of detrital zircons can be a complement to major and trace element geochemistry and Sm-Nd systematics (Díez Fernández et al., 2010; Drost et al., 2004; Linnemann and Romer, 2002; Linnemann et al., 2004). In favourable situations, these data allow to determine the tectonic seting and location of the sedimentary paleo-basins. Sedimentary series formed bygreywackes are particularly useful for these studies since the analysis of immobile elements during post-sedimentary and orogenic processes has been proven to be suitable for constraining the tectonic setting (Bathia and Crook, 1986).

This paper presents a case-study of the metasedimentary rocks from the basal units of the allochthonous complexes of NW Iberia, one of the far-wavelled terranes involved in the Variscan suture that are exposed in southern Europe (Martínez Catalán et al., 2009). The structural and metamorphic characteristics of this crustal-derived terrane indicate 
that it was affected by high-P and low-to-intermediate-T eo-Variscan metamorphism reaching the blueschist and eclogite facies. The origin and geological history of this terrane are still under discussion, and the complex tectonothermal evolution makes difficult to deduce the original setting and provenance of the protoliths of these metasedimentary rock series using raditional techniques. These series include clastic rocks suitable for a provenance study based on geochemical methods. Only few works have applied so far this methodology in the metasedimentary rocks of the Variscan Belt (Fuenlabada et al., 2010; Linnemann et al., 2004, 2007; Ugidos et al., 2003). Moreover, the progressive incoming and comparison of this lind of data may help to correlate the terranes that bound the suture zone of the Variscan Belt.

a

VARISCAN BELT

Allochthonous complexes

Autochthon

External thrust belt and foredeep basin
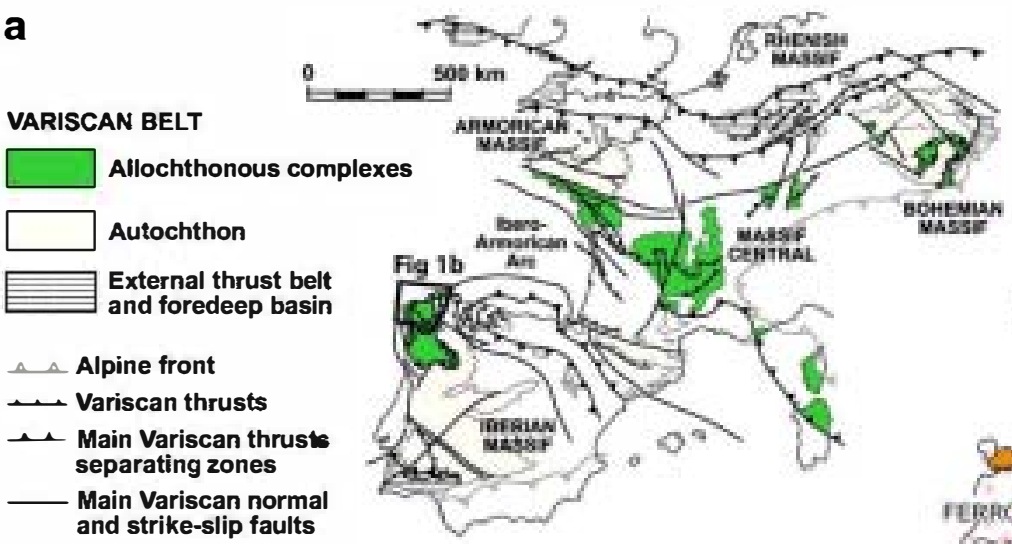

b

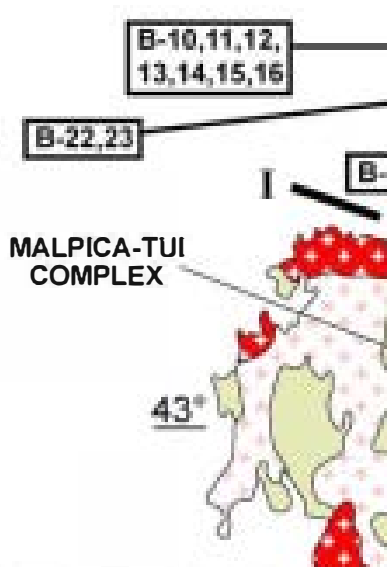

B-19

ALLOCHTHONOUS COMPLEXES:

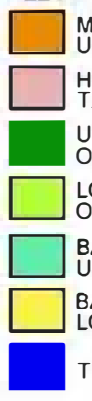

MEDIUM PRESSURE

HIGH PRESSURE AND HIGH
TEMPERATURE UPPER UNITS

UPPER

OPHIOLITIC UNITS

LOWER

OPHIOLITIC UNITS

BASAL UNITS

UPPER SEQUENCE

BASAL UNITS

LOWER SEQUENCE

TECTONIC MÉLANGE

LOWER ALLOCHTHON

AND AUTOCHTHONOUS SEQUENCES

SCHISTOSE DOMAIN AND AUTOCHTHON

VARISCAN GRANITOIDS:

+ POStKInEMATIC / SINKINEMATIC

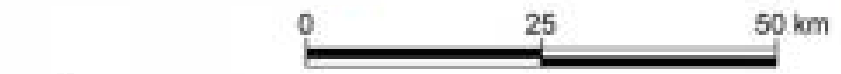

$50 \mathrm{~km}$

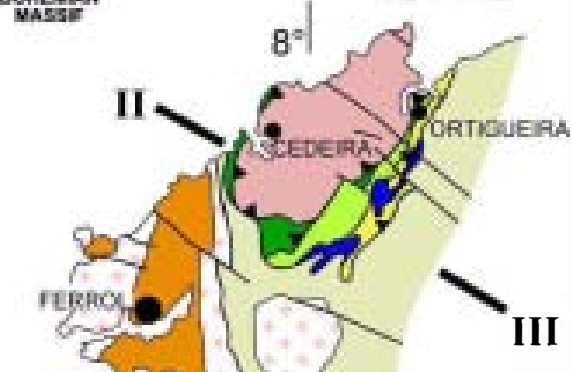

CABO ORTEGAL COMPLEX

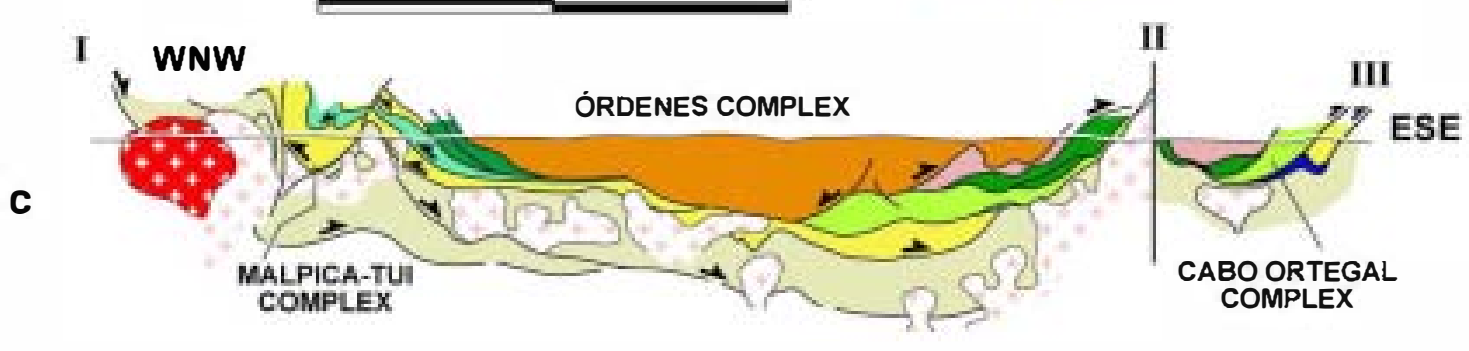

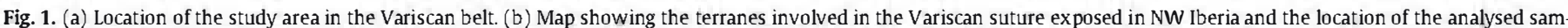

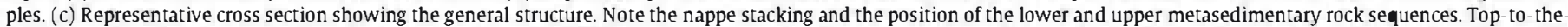

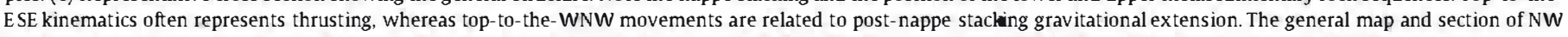
Iberia are based in previous data and maps by Díaz Garcia et al. (1999), Arenas et al. (2009), Martínez Catalán et al. (2009) and Díez Fernández et al. (2010). 


\section{Geological setting}

The NW Iberian section of the Variscan Belt contains a set of allochthonous terranes thrust over an autochthonous domain representing

a

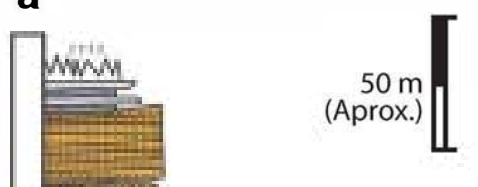

b
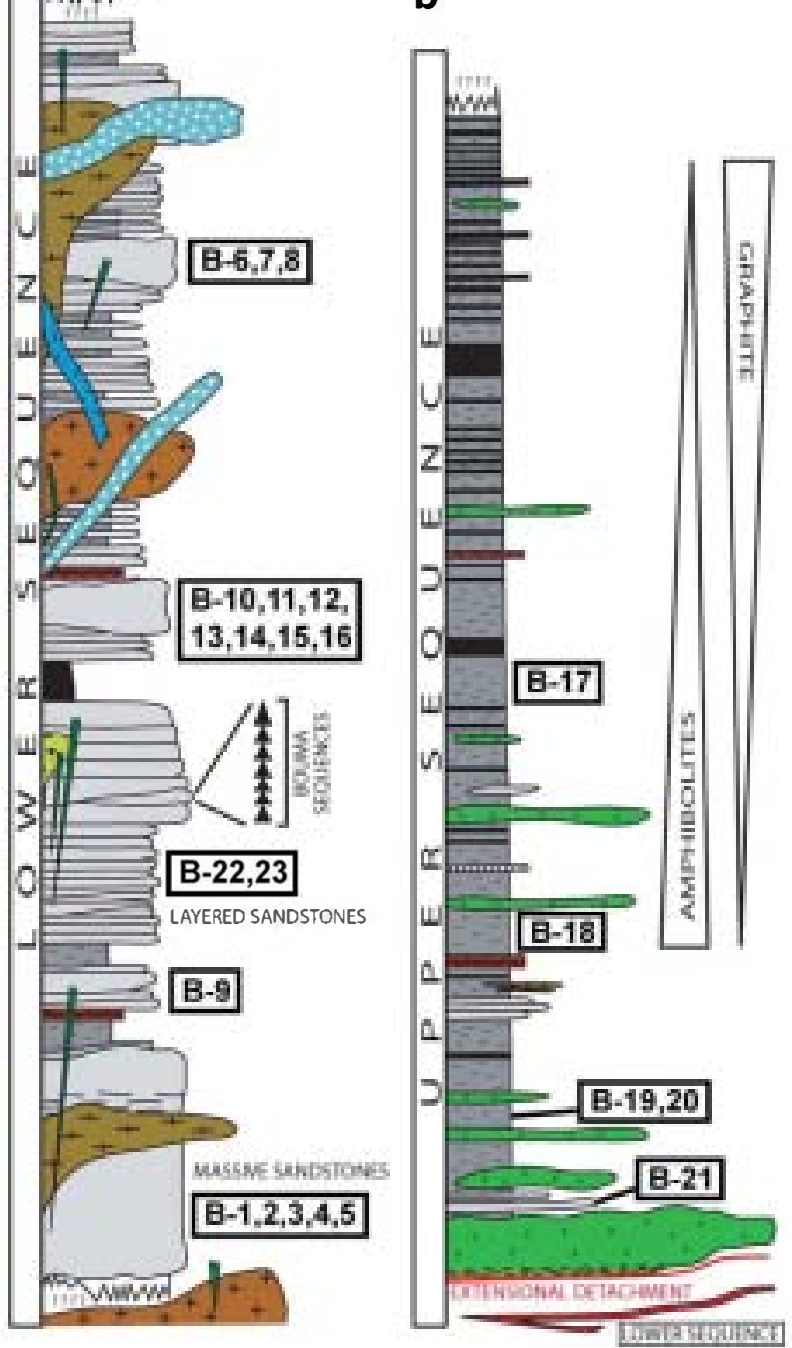

SEDIMENTARY-IGNEOUS / METAMORPHIC LITHOLOGIES

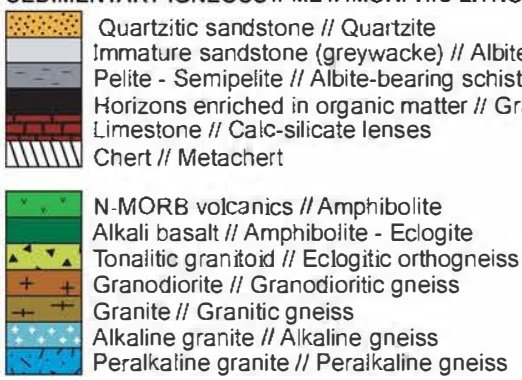

Fig. 2. Idealized stratigraphic columns summarizing the main sedimentological features observed in the lower (a) and upper (b) sequences. The position of the analysed samples is also shown. Due to strong Variscan deformation, the vertical distribution of the facies of the lower sequence shown in this figure is approximate. the margin of Gondwana (Fig. 1a). It is generally accepted that the lowermost allochthonous terrane (Parautochthon), mainly constituted by metasedimentary rocks, represents an external section of the Paleozoic Gondwana margin. However, the interpretation of those terranes located either in the hanging wall or in the footwall to the Variscan suture zone is more complex, as they are probably affected by important displacements and may have an exotic nature (Martínez Catalán et al., 2007, 2009). In the NW Iberian Massif, the suture zone is marked by several ophiolitic units (Fig. 1b and c) different in age and likely related to different geodynamic settings, but considered as a whole formed during different evolutionary stages of the Rheic Ocean. These units were obducted over the most external margin of Gondwana at the onset of the Variscan collision, after closure of this ocean at the end of Devonian times (Arenas et al., 2007; Díaz García et al., 1999; Gómez Barreiro et al., 2010; Sánchez Martínez et al., 2007).

The upper section of the allochthonous pile (upper units) rests over the ophiolites and contains an intricate succession of siliciclastic metasedimentary rocks intruded by large massifs of granitoids and gabbros (Fig. 1b and c). This terrane has a polymetamorphic tectonothermal evolution developed during two consecutive Middle Cambrian and Late Silurian-Middle Devonian events (Abati et al., 1999, 2007; Fernández-Suárez et al., 2002, 2007). The upper units are considered a section of a Cambrian peri-Gondwanan magmatic arc, some of these units show a counter-clockwise P-T evolution associated to magmatic underplating (Abati et al., 2003). In a subsequent event, the arc drifted away from Gondwana leaving the Rheic Ocean at its tail, being accreted to the southern margin of Iaurussia afterwards (Gómez Barreiro et al., 2007). The uppermost sequence of this terrane is constituted by a low grade thick sequence of metagreywackes intruded by a network of diabasic dikes. These metagreywackes are Middle Cambrian in age, and their tectonic setting was revealed using major and trace element geochemistry and Sm-Nd systematics (Fuenlabrada et al., 2010).

The basal units of the allochthonous complexes define a crustal accretionary complex stacked below the ophiolites (Fig. 1c). They are formed by thick metasedimentary rock sequences intruded by calc-alkaline granitoids (c. $493 \mathrm{Ma}$; Abati et al., 2010b; Díez Fernández et al., 2012) and minor alkaline to peralkaline massifs (c. 475-470 Ma; Díez Fernández et al., 2012; Rodríguez Aller, 2005; Rodríguez et al., 2007), both transformed in variably deformed orthogneisses, as well as mafic rocks transformed in amphibolites, blueschists and eclogites (Gil Ibarguchi and Ortega Gironés, 1985). These units are considered to represent a section of the most external margin of Gondwana (Arenas et al., 1986; Díez Fernández et al., 2011; Martínez Catalán et al., 1996). According to the chemical composition of the orthogneisses, the protoliths of the basal units were formed in the context of a peri-Gondwanan magmatic arc (calc-alkaline granitoids), affected by subsequent rifting during the opening of the Rheic Ocean (alkaline-peralkaline granitoids; Abati et al., 2010b). However, any description of the compositional characteristics of the metasedimentary rocks did not exist until now, although these are the oldest lithologies and they can be used to deduce the initial tectonic setting. This margin was subsequently affected by eo-Variscan high-P low-tointermediate-T metamorphism, developed at c. 370 Ma during subduction beneath Laurussia at the onset of the Variscan collision, in the main stage of the Pangea assembly (Abati et al., $2010 \mathrm{~b}$; Rodríguez et al., 2003).

\section{The sedimentary record of the basal units}

Two different and superimposed metasedimentary rock sequences can be distinguished in the basal units. The deformation shows an heterogeneous character with important partitioning, which allows the preservation of low to very low deformed sections, 
sometimes almost intact and even preserving the original sedimentary features (Díez Fernández, 2011). In the low deformed sections, the lower metasedimentary sequence consists of a thick pile of metagreywackes alternating with minor layers of metapelites, graphitic schists, calc-silicate lenses and quartzites (Fig. 2a). The metagreywackes preserve Bouma sequences, crossed bedding, erosive contacts and normal graded bedding. Their protoliths were clastsupported sedimentary rocks containing angular feldspar fragments (the most abundant clasts), quartz and detrital micas in a clay rich matrix with carbonaceous material. No conglomeratic levels have been found. Metapelitic horizons are common, and carbonaceous matter within them can be so abundant as to form graphitic horizons. Quartzite layers occur in the upper part of the sequence (Fig. 2a). The original thickness of the lower sequence cannot be calculated due to the intense ductile deformation accompanying the Variscan subduction and subsequent exhumation, but a minimum present thickness of $4 \mathrm{~km}$ can be estimated, although this value probably represents less than half of the original thickness. The maximum depositional age, as revealed by the detrital zircon input, is latest Neoproterozoic (c. $560 \mathrm{Ma}$; Díez Fernández et al., 2010).

The upper sequence appears strongly deformed. No significant deformation partitioning associated with the subduction-exhumation process can be seen in this series during the subduction-exhumation process. This sequence consists of mica schists alternating with minor lenses of amphibolites, graphite schists, metacherts, calc-silicate lenses, metagreywackes and quartzites (Fig. 2b). The maximum depositional age of this sequence is Late Cambrian (c. $500 \mathrm{Ma}$; Diez Fernández et al., 2010).

\section{Whole rock geochemistry}

The chemical composition of sedimentary rocks depends on numerous factors, including the nature of the source areas and the subsequent processes affecting them, such as weathering, diagenesis or metamorphism. Likewise, the abundance of some elements, such as rare earth elements (REE), $\mathrm{Hf}, \mathrm{Ti}, \mathrm{Cr}, \mathrm{Co}, \mathrm{Zr}, \mathrm{Nb}, \mathrm{Ta}, \mathrm{Y}$, Th and $\mathrm{Sc}$, is preserved in sedimentary rocks in spite of the weathering processes. These elements have very low residence times in oceanic waters, being transferred almost quantitatively to sedimentary rocks. Thus, they provide excellent discriminating factors for determining the provenance and tectonic setting of sedimentary rocks (Bathia, 1983; Bathia and Crook, 1986; Hegde and Chavadi, 2009; Roser and Korsch, 1986, 1988; Taylor and McLennan, 1985). The effects of homogenization in sedimentary processes result in a relatively uniform distribution of REE in detrital rocks, whose pattern reflects the REE abundance in the upper crust. Moreover, the Th/Sc, $\mathrm{La} / \mathrm{Sc}, \mathrm{Ti} / \mathrm{Zr}$, $\mathrm{Zr} / \mathrm{Sc}$ and $\mathrm{La} / \mathrm{Th}$ ratios has been frequently used to investigate provenance according to the different compatibility of these elements during magmatic crystallization (Mclennan et al., 1993), and also in relation to the PAAS abundances (Post Archean Australian Shale; Taylor and McLennan, 1985).

Twenty three samples from the sedimentary series that constitutes the basal units of the allochthonous complexes of NW Iberia were collected in order to study their geochemistry, provenance and tectonic setting. Samples were collected mainly along the northern sector of the Malpica-Tui Complex although a few samples were taken in the NW part of the Órdenes Complex (see location of the samples in Figs. 1b and 2). Eighteen samples belong to the lower sequence and they include weakly sheared metagreywackes and some albite schists developed after intense deformation and metamorphism of the greywackic types. Five samples represent the upper sequence including the Cean schists (Malpica-Tui Complex) and the Santiago schists (NW Órdenes Complex).

Sample preparation was carried out at Universidad Complutense de Madrid, and whole rock major and trace elements analyses were performed at Activation Laboratories Ltd (Actlabs) in Canada. Fusion with lithium metaborate/tetraborate was used for sample digestion, and the analytical techniques for major and trace element determination were ICP-OES and ICP-MS, respectively. The chemical analyses results are shown in Tables 1 and 2 .

\subsection{Composition and classification}

\subsubsection{Lower sequence}

The greywackes and albitic schists show a homogeneous major element composition (Table 1). The $\mathrm{SiO}_{2}$ content shows the largest variation (60.3-74.9wt.\%), with average value of $70.65 w t . \%$. Only 4 samples have $\mathrm{SiO}_{2}$ contents lower than $70 \mathrm{wt} . \%$ (B-3, B-5, B-9, B-23), mainly compensated by larger values of $\mathrm{Al}_{2} \mathrm{O}_{3}$ and $\mathrm{MgO}$. The $\mathrm{Na}_{2} \mathrm{O}$ content is relatively high and homogeneous (2.5-4.1 wt.\%; average $2.9 \mathrm{wt} . \%)$, and also can be considered homogeneous the content in $\mathrm{K}_{2} \mathrm{O}$ (2.0-3.4wt.\%; average 2.6 wt.\%) and $\mathrm{MgO}$ (1.1-3.4 wt.\%; average $1.7 \mathrm{wt} . \%)$. The compositional range of the other major elements is: $\mathrm{CaO}$ (0.5-2.9 wt.\%; average $1.0 \mathrm{wt} . \%), \mathrm{Al}_{2} \mathrm{O}_{3}$ (11.0-17.8 wt.\%; average $13.3 \mathrm{wt} . \%), \mathrm{Fe}_{2} \mathrm{O}_{3}$ (3.3-6.8 wt.\%; average 4.32 wt.\%), $\mathrm{MnO}$ (0.030.14 wt.\%), $\mathrm{TiO}_{2}\left(0.50-0.83\right.$ wt.\%) and $\mathrm{P}_{2} \mathrm{O}_{5}$ (0.13-0.20 wt.\%). $\mathrm{SiO}_{2}$ shows marked negative correlation with $\mathrm{Al}_{2} \mathrm{O}_{3}, \mathrm{Fe}_{2} \mathrm{O}_{3}, \mathrm{MnO}$ and $\mathrm{MgO}$, and moderate negative correlation with $\mathrm{CaO}, \mathrm{Na}_{2} \mathrm{O}, \mathrm{K}_{2} \mathrm{O}, \mathrm{TiO}_{2}$ and $\mathrm{P}_{2} \mathrm{O}_{5}$. The relatively low $\mathrm{SiO}_{2} / \mathrm{Al}_{2} \mathrm{O}_{3}$ ratio of these rocks indicates an immature character, as confirmed by the $\mathrm{Al}_{2} \mathrm{O}_{3} / \mathrm{Na}_{2} \mathrm{O}$ (3.6-5.8) and $\mathrm{Al}_{2} \mathrm{O}_{3}$ / $\mathrm{TiO}_{2}$ (16.8-23.9) ratios, which lay within the typical range of the upper continental crust (3.9 and 30.4, respectively; Taylor and McLennam, 1985).

The $\mathrm{Fe}_{2} \mathrm{O}_{3} / \mathrm{K}_{2} \mathrm{O}$ ratio can be applied to distinguish between mature and immature compositions of unconsolidated fine- to coarsegrained sediments (particularly in arkoses). According to the chemical classification diagram published by Herron (1988), most of the protoliths of the metasedimentary rocks of the lower sequence can be classified as greywackes, but some few samples (Xareira metagreywackes; Diez Fernández, 2011) plot in the boundary with the litharenite field (Fig. 3a). According to the $\mathrm{K}_{2} \mathrm{O} / \mathrm{Na}_{2} \mathrm{O}$ ratio, most of the analysed rocks have quartz-intermediate compositions (Crook, 1974).

Table 2 shows the results of the REE analyses. The samples have similar REE contents, with $\Sigma$ REE values ranging between $112 \mathrm{ppm}$ (sample B-22, with marked depletion in IREE) and $213 \mathrm{ppm}$ (the highest values in some samples with the highest contents in La and Ce). The samples also show similar chondrite-normalized (Nakamura, 1974) fractionation patterns (Fig. 3b), with a $(\mathrm{Ia} / \mathrm{Yb})_{N}$ ratio ranging between 7.1 and 14.4. The IREE (Ia-Sm) show a moderate enrichment in relation to the HREE, which display almost flat pattems with $(\mathrm{Gd} / \mathrm{Yb})_{\mathrm{N}}$ ratios ranging between 1.0 and 1.7. All the samples show slight but significant negative Eu anomaly, variable between 0.57 and 0.77 (calculated according to Taylor and Mcl.ennan, 1985). Eu anomalies in sedimentary rocks are usually interpreted as inheritance from the igneous rock source. Despite some differences in abundance between samples, the REE patterns of the greywackes are similar to those of PAAS, which are considered representative of upper continental crust.

The analysed samples show an average $\mathrm{Th} / \mathrm{Sc}$ value of 1.2 , larger than the PAAS value $(\mathrm{Th} / \mathrm{Sc}=0.9)$ and probably suggesting a source area with predominance of felsic rocks. This interpretation is also based in the high Ia contents in relation to Sc, with average La/ $\mathrm{Sc}=4.27$ above the PAAS ratio $(\mathrm{La} / \mathrm{Sc}=2.4)$. The same interpretation can be obtained from the $\mathrm{Ti} / \mathrm{Zr}$ and $\mathrm{Zr} / \mathrm{Sc}$ ratios, respectively lower and higher than PAAS, and also from the low contents in $\mathrm{Cr}$ (average $73 \mathrm{ppm}$ ) and $\mathrm{Ni}$ (average $26 \mathrm{ppm}$ ). Moreover, the La/Th ratio ranging from 2.4 to 4.7 with an average value of 3.5, higher than PAAS (2.6), also suggests the abundance of felsic rocks in the source areas. The relatively high $\mathrm{Hf}$ contents (average 6.2; PAAS $=5$ ) can be also considered an indication of source areas located in the surrounding area of a passive margin (Hegde and Chavadi, 2009). 
Table 1

Whole rock major and trace element data of metasedimentary rocks.

\begin{tabular}{|c|c|c|c|c|c|c|c|c|c|c|c|}
\hline Sample & B-1 & B-2 & B-3 & B-4 & B-5 & B-6 & B-7 & B-8 & B-9 & B-10 & B-11 \\
\hline $\mathrm{SiO}_{2}$ & 73.83 & 73.94 & 60.34 & 71.77 & 64.50 & 70.81 & 70.51 & 70.14 & 61.40 & 73.12 & 74.40 \\
\hline $\mathrm{Al}_{2} \mathrm{O}_{3}$ & 12.62 & 12.36 & 16.77 & 12.97 & 15.85 & 13.22 & 13.52 & 13.52 & 17.85 & 12.06 & 11.24 \\
\hline $\mathrm{Fe}_{2} \mathrm{O}_{3}$ & 3.36 & 3.38 & 6.82 & 4.46 & 5.60 & 4.00 & 4.29 & 4.85 & 5.92 & 3.46 & 3.96 \\
\hline $\mathrm{MnO}$ & 0.049 & 0.047 & 0.077 & 0.058 & 0.068 & 0.048 & 0.049 & 0.055 & 0.149 & 0.033 & 0.042 \\
\hline $\mathrm{MgO}$ & 1.21 & 1.13 & 3.38 & 1.72 & 2.45 & 1.55 & 1.74 & 1.80 & 2.47 & 1.24 & 1.23 \\
\hline $\mathrm{CaO}$ & 1.83 & 1.50 & 2.85 & 1.55 & 1.33 & 0.88 & 0.63 & 0.76 & 1.63 & 0.61 & 0.52 \\
\hline $\mathrm{Na}_{2} \mathrm{O}$ & 3.09 & 2.91 & 4.13 & 3.35 & 2.79 & 3.64 & 2.73 & 3.07 & 2.56 & 2.86 & 2.79 \\
\hline $\mathrm{K}_{2} \mathrm{O}$ & 2.01 & 2.10 & 2.48 & 2.15 & 3.42 & 2.02 & 2.66 & 2.66 & 2.88 & 2.94 & 2.52 \\
\hline $\mathrm{TiO}_{2}$ & 0.557 & 0.563 & 0.834 & 0.542 & 0.725 & 0.576 & 0.583 & 0.768 & 0.777 & 0.509 & 0.659 \\
\hline $\mathrm{P}_{2} \mathrm{O}_{5}$ & 0.15 & 0.17 & 0.16 & 0.13 & 0.16 & 0.14 & 0.15 & 0.20 & 0.18 & 0.13 & 0.15 \\
\hline $\mathrm{LOI}^{\mathrm{a}}$ & 0.9 & 0.8 & 1.4 & 0.8 & 1.5 & 1.8 & 2.5 & 1.9 & 2.4 & 1.4 & 1.8 \\
\hline Total & 99.62 & 98.93 & 99.19 & 99.51 & 98.36 & 98.71 & 99.30 & 99.75 & 98.23 & 98.35 & 99.25 \\
\hline Sc & 8 & 8 & 19 & 9 & 14 & 9 & 10 & 11 & 15 & 7 & 8 \\
\hline V & 58 & 58 & 133 & 62 & 92 & 65 & 65 & 81 & 105 & 56 & 71 \\
\hline $\mathrm{Cr}$ & 80 & 60 & 100 & 80 & 90 & 60 & 60 & 80 & 90 & 70 & 140 \\
\hline Co & 15 & 12 & 26 & 15 & 15 & 7 & 9 & 9 & 11 & 10 & 11 \\
\hline $\mathrm{Ni}$ & 30 & 20 & 50 & 30 & 40 & 20 & 20 & 20 & 30 & 30 & 30 \\
\hline $\mathrm{Cu}$ & 20 & $<10$ & 20 & 30 & 20 & $<10$ & $<10$ & $<10$ & 40 & 20 & 20 \\
\hline $2 n$ & 50 & 50 & 90 & 80 & 80 & 50 & 50 & 60 & 90 & 50 & 70 \\
\hline $\mathrm{Ga}$ & 15 & 14 & 23 & 15 & 20 & 16 & 16 & 17 & 22 & 14 & 14 \\
\hline $\mathrm{Rb}$ & 73 & 79 & 99 & 89 & 131 & 83 & 89 & 100 & 87 & 94 & 88 \\
\hline Sr & 153 & 121 & 207 & 164 & 133 & 164 & 125 & 135 & 198 & 144 & 117 \\
\hline Y & 22.9 & 23.1 & 28.9 & 22.9 & 22.7 & 20.2 & 17.9 & 25.8 & 32.1 & 17.8 & 21.4 \\
\hline $\mathrm{Zr}$ & 268 & 315 & 167 & 166 & 200 & 250 & 182 & 430 & 234 & 214 & 381 \\
\hline $\mathrm{Nb}$ & 11.2 & 9.7 & 12.9 & 10.8 & 13.7 & 10.1 & 9.0 & 12.6 & 14.0 & 9.4 & 12.4 \\
\hline Cs & 5.4 & 5.8 & 12.1 & 8.1 & 7.1 & 5.2 & 5.0 & 6.4 & 9.6 & 4.9 & 4.7 \\
\hline $\mathrm{Ba}$ & 584 & 453 & 963 & 466 & 716 & 629 & 881 & 903 & 999 & 1004 & 838 \\
\hline $\mathrm{Hf}$ & 6.5 & 7.2 & 4.3 & 4.1 & 5.0 & 5.9 & 4.2 & 10.0 & 5.6 & 5.1 & 9 \\
\hline $\mathrm{Ta}$ & 0.94 & 0.91 & 1.11 & 0.90 & 1.19 & 0.92 & 0.92 & 1.15 & 1.33 & 0.84 & 1.05 \\
\hline $\mathrm{Pb}$ & 28 & 18 & 32 & 18 & 12 & 9 & 7 & 11 & 15 & 23 & 22 \\
\hline Th & 9.88 & 11.20 & 11.10 & 7.92 & 10.50 & 10.70 & 9.36 & 14.30 & 12.90 & 9.26 & 14.3 \\
\hline $\mathrm{U}$ & 2.92 & 2.93 & 3.26 & 2.42 & 3.13 & 2.59 & 2.36 & 3.55 & 3.97 & 2.63 & 3.43 \\
\hline $\mathrm{Rb} / \mathrm{Sr}$ & 0.48 & 0.65 & 0.48 & 0.54 & 0.98 & 0.51 & 0.71 & 0.74 & 0.44 & 0.65 & 0.75 \\
\hline $\mathrm{Ti} / \mathrm{Zr}$ & 12.46 & 10.71 & 29.94 & 19.57 & 21.73 & 13.81 & 19.20 & 10.71 & 19.91 & 14.26 & 10.7 \\
\hline $\mathrm{La} / \mathrm{Sc}$ & 4.91 & 5.46 & 2.23 & 4.11 & 2.81 & 4.28 & 2.64 & 4.54 & 3.85 & 4.94 & 6.43 \\
\hline $\mathrm{La} / \mathrm{Y}$ & 1.72 & 1.89 & 1.46 & 1.62 & 1.73 & 1.91 & 1.47 & 1.93 & 1.80 & 1.94 & 2.4 \\
\hline $\mathrm{La} / \mathrm{Th}$ & 3.98 & 3.90 & 3.81 & 4.67 & 3.74 & 3.60 & 2.82 & 3.49 & 4.48 & 3.74 & 3.59 \\
\hline $\mathrm{Sc} / \mathrm{Cr}$ & 0.10 & 0.13 & 0.19 & 0.11 & 0.16 & 0.15 & 0.17 & 0.14 & 0.17 & 0.10 & 0.06 \\
\hline $\mathrm{Th} / \mathrm{Sc}$ & 1.24 & 1.40 & 0.58 & 0.88 & 0.75 & 1.19 & 0.94 & 1.30 & 0.86 & 1.32 & 1.79 \\
\hline
\end{tabular}

Oxides are in weight percent (wt.\%).

Trace elements are in parts per million ( $\mathrm{ppm}$ ).

The element concentrations expressed with the $<$ sign are below detection limit.

a Loss on ignition.

Table 2

Whole rock rare earth element data of metasedimentary rocks.

\begin{tabular}{|c|c|c|c|c|c|c|c|c|c|c|c|}
\hline \multicolumn{12}{|c|}{ Lower sedimentary sequence } \\
\hline & B-1 & B-2 & B-3 & B-4 & B-5 & B-6 & B-7 & B-8 & B-9 & B-10 & B-11 \\
\hline La & 39.3 & 43.7 & 42.3 & 37 & 39.3 & 38.5 & 26.4 & 49.9 & 57.8 & 34.6 & 51.4 \\
\hline $\mathrm{Ce}$ & 71.5 & 80.5 & 79.4 & 65.9 & 73.7 & 59.3 & 48.3 & 87.6 & 70.6 & 61.7 & 94.5 \\
\hline $\operatorname{Pr}$ & 8.05 & 9.03 & 9.23 & 7.47 & 8.41 & 8.14 & 5.97 & 10.7 & 11.4 & 7.2 & 10.4 \\
\hline Nd & 27.9 & 32.3 & 32.5 & 26.6 & 29.6 & 29.4 & 21.7 & 38.1 & 41.5 & 24.7 & 34.5 \\
\hline Sm & 5.28 & 6.11 & 6.66 & 5.13 & 5.88 & 5.48 & 4.39 & 7.04 & 7.95 & 4.61 & 6.07 \\
\hline Eu & 1.10 & 1.10 & 1.29 & 1.15 & 1.16 & 1.08 & 0.92 & 1.27 & 1.64 & 0.95 & 0.98 \\
\hline Gd & 4.46 & 4.74 & 5.88 & 4.34 & 4.95 & 4.27 & 3.38 & 5.33 & 6.58 & 3.62 & 4.64 \\
\hline $\mathrm{Tb}$ & 0.73 & 0.76 & 0.97 & 0.71 & 0.78 & 0.67 & 0.56 & 0.86 & 1.03 & 0.59 & 0.71 \\
\hline Dy & 4.01 & 4.3 & 5.35 & 3.85 & 4.19 & 3.81 & 3.33 & 4.89 & 5.83 & 3.27 & 3.97 \\
\hline Ho & 0.79 & 0.83 & 1.01 & 0.77 & 0.82 & 0.74 & 0.66 & 0.95 & 1.12 & 0.62 & 0.78 \\
\hline Er & 2.3 & 2.41 & 2.97 & 2.24 & 2.43 & 2.18 & 1.95 & 2.8 & 3.32 & 1.85 & 2.27 \\
\hline Tm & 0.373 & 0.372 & 0.466 & 0.345 & 0.380 & 0.328 & 0.309 & 0.442 & 0.512 & 0.309 & 0.364 \\
\hline $\mathrm{Yb}$ & 2.4 & 2.5 & 2.9 & 2.2 & 2.5 & 2.2 & 2.0 & 2.9 & 3.3 & 2.0 & 2.38 \\
\hline Lu & 0.344 & 0.347 & 0.416 & 0.308 & 0.358 & 0.322 & 0.282 & 0.423 & 0.469 & 0.287 & 0.364 \\
\hline$\Sigma \mathrm{REE}$ & 168.50 & 188.97 & 191.31 & 158.01 & 174.42 & 156.44 & 120.16 & 213.18 & 213.05 & 146.31 & 213.33 \\
\hline $\mathrm{Eu} / \mathrm{Eu}^{*}$ & 0.70 & 0.63 & 0.63 & 0.75 & 0.66 & 0.69 & 0.73 & 0.64 & 0.70 & 0.72 & 0.57 \\
\hline$(\mathrm{La} / \mathrm{Sm}) \mathrm{N}$ & 4.59 & 4.41 & 3.92 & 4.45 & 4.12 & 4.33 & 3.71 & 4.37 & 4.49 & 4.63 & 5.22 \\
\hline$(\mathrm{Gd} / \mathrm{Yb})_{\mathrm{N}}$ & 1.51 & 1.53 & 1.63 & 1.57 & 1.6 & 1.53 & 1.34 & 1.48 & 1.59 & 1.44 & 1.55 \\
\hline$(\mathrm{La} / \mathrm{Yb})_{\mathrm{N}}$ & 11.14 & 11.83 & 9.86 & 11.25 & 10.68 & 11.6 & 8.78 & 11.63 & 11.71 & 11.57 & 14.44 \\
\hline
\end{tabular}

Rare earth elements data in parts per million (ppm). 


\begin{tabular}{|c|c|c|c|c|c|c|c|c|c|c|c|}
\hline \multirow[b]{2}{*}{ B-12 } & \multirow[b]{2}{*}{ B-13 } & \multirow[b]{2}{*}{ B-14 } & \multirow[b]{2}{*}{ B-15 } & \multirow[b]{2}{*}{ B-16 } & \multirow[b]{2}{*}{ B-22 } & \multirow[b]{2}{*}{ B-23 } & \multicolumn{5}{|c|}{ Upper sedimentary sequence } \\
\hline & & & & & & & B-17 & B-18 & B-19 & B-20 & B-21 \\
\hline 73.36 & 73.23 & 74.89 & 72.50 & 73.84 & 73.27 & 65.94 & 59.77 & 59.59 & 57.63 & 58.87 & 72.9 \\
\hline 11.73 & 12.05 & 11.08 & 12.55 & 11.82 & 12.83 & 16.05 & 19.82 & 19.59 & 19.59 & 18.86 & 14.58 \\
\hline 3.77 & 3.67 & 3.74 & 3.73 & 3.57 & 3.72 & 5.62 & 7.73 & 7.51 & 8.33 & 8.58 & 1.53 \\
\hline 0.038 & 0.033 & 0.039 & 0.036 & 0.032 & 0.05 & 0.042 & 0.511 & 0.222 & 0.272 & 0.414 & 0.025 \\
\hline 1.32 & 1.32 & 1.23 & 1.38 & 1.30 & 1.47 & 2.31 & 2.23 & 1.97 & 2.50 & 1.91 & 0.28 \\
\hline 0.64 & 0.49 & 0.71 & 0.58 & 0.62 & 1 & 0.78 & 0.32 & 0.36 & 0.45 & 1.01 & 0.33 \\
\hline 2.74 & 2.65 & 3.00 & 2.78 & 3.02 & 3.3 & 2.51 & 0.70 & 0.66 & 1.05 & 1.06 & 2.61 \\
\hline 2.78 & 2.85 & 2.19 & 3.09 & 2.45 & 2.32 & 3.2 & 3.77 & 4.04 & 3.52 & 4.48 & 4.77 \\
\hline 0.581 & 0.539 & 0.660 & 0.549 & 0.533 & 0.566 & 0.683 & 0.817 & 0.891 & 0.926 & 0.719 & 0.205 \\
\hline 0.14 & 0.14 & 0.14 & 0.14 & 0.15 & 0.15 & 0.13 & 0.13 & 0.14 & 0.18 & 0.09 & 0.27 \\
\hline 1.3 & 1.4 & 1.6 & 1.5 & 1.2 & 1.12 & 2.48 & 3.5 & 3.7 & 4.1 & 3.1 & 1.89 \\
\hline 98.37 & 98.33 & 99.26 & 98.80 & 98.52 & 99.8 & 99.74 & 99.27 & 98.62 & 98.55 & 99.05 & 99.39 \\
\hline 8 & 8 & 8 & 8 & 8 & 8 & 13 & 19 & 20 & 20 & 20 & 3 \\
\hline 61 & 60 & 71 & 61 & 60 & 64 & 96 & 164 & 132 & 124 & 131 & 8 \\
\hline 60 & 60 & 60 & 50 & 60 & 50 & 70 & 100 & 90 & 140 & 100 & $<20$ \\
\hline 8 & 9 & 8 & 11 & 14 & 13 & 12 & 16 & 15 & 25 & 25 & 3 \\
\hline$<20$ & $<20$ & $<20$ & 20 & $<20$ & 20 & 30 & 50 & 40 & 70 & 50 & $<20$ \\
\hline 10 & 10 & 10 & 20 & 10 & 10 & 20 & 20 & 20 & 400 & 140 & 60 \\
\hline 30 & 40 & 50 & 50 & 60 & 50 & 70 & 110 & 100 & 130 & 100 & 100 \\
\hline 13 & 14 & 12 & 15 & 13 & 15 & 20 & 26 & 26 & 25 & 24 & 24 \\
\hline 94 & 100 & 85 & 105 & 94 & 84 & 114 & 144 & 162 & 153 & 144 & 408 \\
\hline 136 & 115 & 133 & 123 & 146 & 163 & 158 & 118 & 96 & 122 & 121 & 28 \\
\hline 20.4 & 18.7 & 20.8 & 20.9 & 22.2 & 19.6 & 27.1 & 27.4 & 28.6 & 30.9 & 24.5 & 8.7 \\
\hline 278 & 238 & 390 & 272 & 267 & 248 & 189 & 119 & 172 & 121 & 110 & 68 \\
\hline 9.5 & 8.4 & 10.7 & 8.9 & 9.9 & 9.1 & 11.5 & 16.1 & 13.8 & 16.2 & 12.4 & 16.1 \\
\hline 4.7 & 5.2 & 4.5 & 5.1 & 5.7 & 5.2 & 8.9 & 4.9 & 6 & 8.8 & 5.3 & 30 \\
\hline 966 & 1039 & 765 & 1132 & 828 & 651 & 773 & 609 & 657 & 541 & 677 & 62 \\
\hline 6.6 & 5.4 & 8.9 & 6.5 & 6.3 & 5.8 & 4.6 & 3.1 & 4.2 & 3.2 & 2.9 & 2.1 \\
\hline 0.96 & 0.87 & 1.04 & 0.92 & 0.91 & 0.96 & 1.09 & 1.26 & 1.32 & 1.24 & 1.05 & 2.64 \\
\hline 18 & 14 & 20 & 17 & 18 & 13 & 15 & 33 & 27 & 32 & 19 & 33 \\
\hline 12.2 & 9.47 & 13.3 & 12 & 15.2 & 10 & 10.9 & 14.5 & 16.7 & 14.2 & 12.5 & 14.7 \\
\hline 2.88 & 2.55 & 3.14 & 2.8 & 2.78 & 2.23 & 2.83 & 2.76 & 1.98 & 2.47 & 3.78 & 10.5 \\
\hline 0.69 & 0.87 & 0.64 & 0.85 & 0.64 & 0.52 & 0.72 & 1.22 & 1.69 & 1.25 & 1.19 & 14.57 \\
\hline 12.53 & 13.58 & 10.15 & 12.1 & 11.97 & 13.68 & 21.66 & 41.16 & 31.06 & 45.88 & 39.19 & 18.07 \\
\hline 5.06 & 4.48 & 5.13 & 5.08 & 5.00 & 2.95 & 2.93 & 2.84 & 2.58 & 2.56 & 0.81 & 7.1 \\
\hline 1.99 & 1.91 & 1.97 & 1.94 & 1.8 & 1.20 & 1.41 & 1.97 & 1.8 & 1.66 & 0.66 & 2.45 \\
\hline 3.32 & 3.78 & 3.08 & 3.38 & 2.63 & 2.36 & 3.50 & 3.72 & 3.09 & 3.61 & 1.29 & 1.45 \\
\hline 0.13 & 0.13 & 0.13 & 0.16 & 0.13 & 0.16 & 0.19 & 0.19 & 0.22 & 0.14 & 0.20 & \\
\hline 1.53 & 1.18 & 1.66 & 1.5 & 1.9 & 1.25 & 0.84 & 0.76 & 0.84 & 0.71 & 0.63 & 4.9 \\
\hline
\end{tabular}

\begin{tabular}{|c|c|c|c|c|c|c|c|c|c|c|c|}
\hline \multirow[b]{2}{*}{ B-12 } & \multirow[b]{2}{*}{ B-13 } & \multirow[b]{2}{*}{ B-14 } & \multirow[b]{2}{*}{ B-15 } & \multirow[b]{2}{*}{ B-16 } & \multirow[b]{2}{*}{ B-22 } & \multirow[b]{2}{*}{ B-23 } & \multicolumn{5}{|c|}{ Upper sedimentary sequence } \\
\hline & & & & & & & B-17 & B-18 & B-19 & B-20 & B-21 \\
\hline 40.5 & 35.8 & 41.0 & 40.6 & 40.0 & 23.6 & 38.1 & 53.9 & 51.6 & 51.2 & 16.1 & 21.3 \\
\hline 76.1 & 66.0 & 79.0 & 74.3 & 74.8 & 47.2 & 72.7 & 108.0 & 99.9 & 93.8 & 39.9 & 46.8 \\
\hline 8.6 & 7.5 & 8.9 & 8.4 & 8.3 & 5.34 & 8.61 & 10.6 & 11.1 & 10.5 & 3.6 & 5.84 \\
\hline 31.1 & 26.4 & 31.1 & 29.8 & 29.4 & 18.9 & 31.4 & 35.5 & 39.1 & 37.4 & 12.3 & 20.2 \\
\hline 5.83 & 4.80 & 6.05 & 5.47 & 5.58 & 3.65 & 6.47 & 6.44 & 7.54 & 7.36 & 2.37 & 4.6 \\
\hline 1.08 & 0.97 & 1.15 & 1.14 & 1.25 & 0.744 & 1.31 & 1.35 & 1.39 & 1.53 & 0.55 & 0.247 \\
\hline 4.54 & 3.67 & 4.62 & 4.24 & 4.48 & 2.9 & 5.12 & 5.32 & 5.71 & 6.13 & 2.44 & 3.09 \\
\hline 0.68 & 0.59 & 0.69 & 0.68 & 0.70 & 0.53 & 0.83 & 0.92 & 0.93 & 0.99 & 0.57 & 0.47 \\
\hline 3.82 & 3.39 & 3.92 & 3.85 & 3.98 & 3.26 & 4.88 & 5.35 & 5.44 & 5.61 & 4.04 & 2.08 \\
\hline 0.74 & 0.67 & 0.75 & 0.76 & 0.79 & 0.67 & 0.92 & 1.04 & 1.03 & 1.09 & 0.88 & 0.3 \\
\hline 2.15 & 1.98 & 2.15 & 2.26 & 2.29 & 2.09 & 2.61 & 3.02 & 2.93 & 3.15 & 2.66 & 0.71 \\
\hline 0.331 & 0.308 & 0.329 & 0.348 & 0.355 & 0.326 & 0.384 & 0.466 & 0.446 & 0.489 & 0.399 & 0.099 \\
\hline 2.26 & 2.07 & 2.18 & 2.33 & 2.36 & 2.23 & 2.54 & 2.92 & 2.77 & 3.06 & 2.55 & 0.56 \\
\hline 0.342 & 0.313 & 0.327 & 0.348 & 0.353 & 0.316 & 0.357 & 0.396 & 0.38 & 0.429 & 0.363 & 0.072 \\
\hline 178.09 & 154.47 & 182.20 & 174.51 & 174.60 & 111.76 & 176.23 & 235.22 & 230.27 & 222.74 & 88.69 & 106.37 \\
\hline 0.65 & 0.71 & 0.67 & 0.73 & 0.77 & 0.70 & 0.70 & 0.71 & 0.65 & 0.7 & 0.7 & 0.20 \\
\hline 4.29 & 4.6 & 4.18 & 4.58 & 4.42 & 3.99 & 3.63 & 5.16 & 4.22 & 4.29 & 4.19 & 2.86 \\
\hline 1.60 & 1.41 & 1.69 & 1.45 & 1.51 & 1.04 & 1.61 & 1.45 & 1.64 & 1.60 & 0.76 & 4.4 \\
\hline 11.98 & 11.56 & 12.58 & 11.65 & 11.33 & 7.08 & 10.03 & 12.34 & 12.46 & 11.19 & 4.22 & 25.43 \\
\hline
\end{tabular}




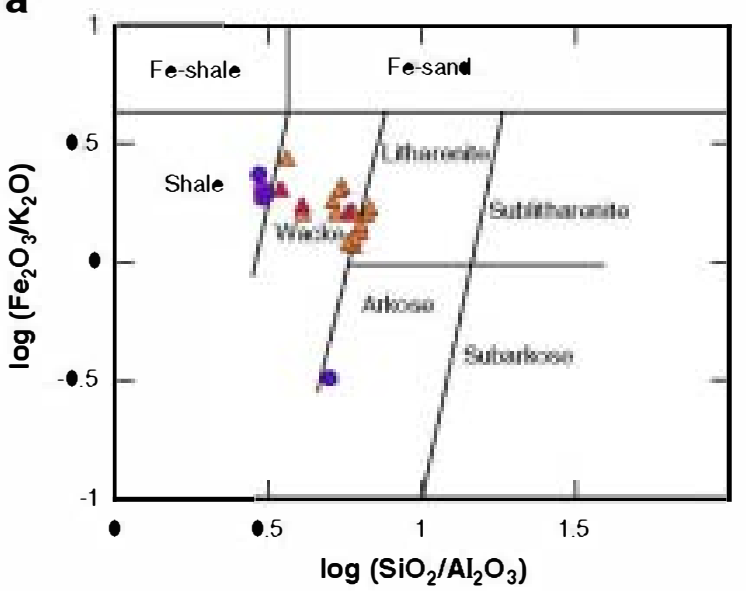

b

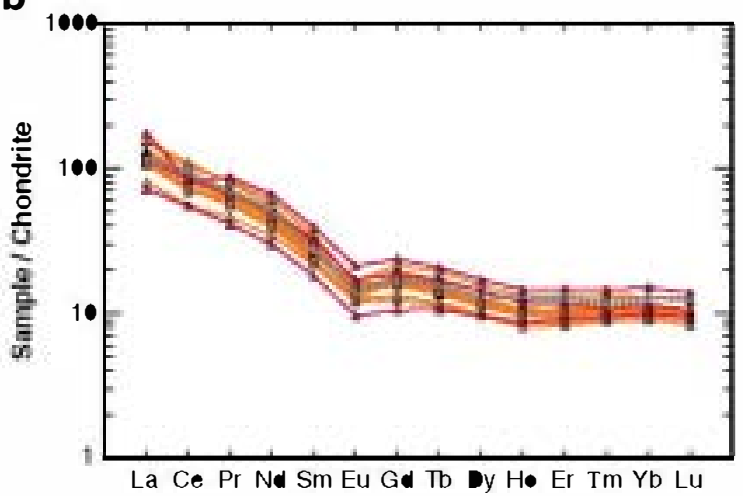

C

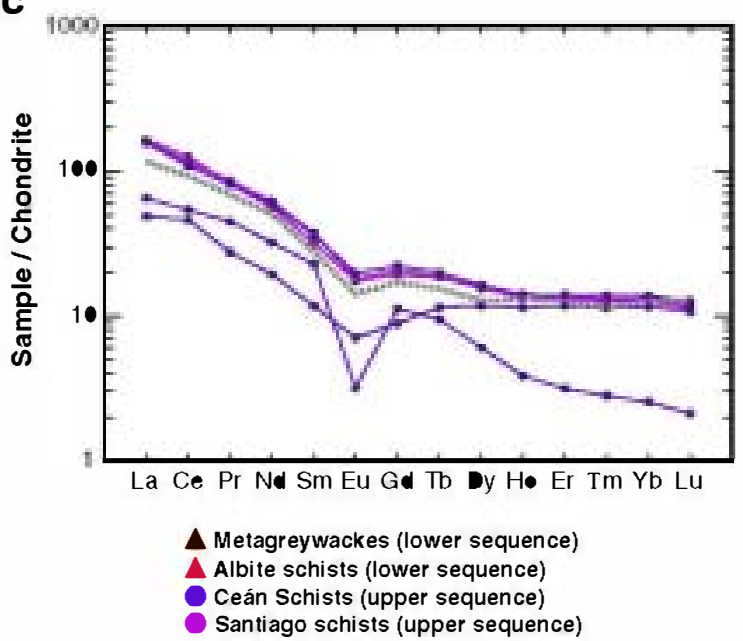

Fig. 3. Chemical diagrams for the metasedimentary rocks from the basal units of NW Iberian Massif. (a) Classification diagram (Herron, 1988). (b, c) Chondritenormalized rare earth elements plots for the metasedimentary rocks of the lower and upper sequences, respectively; the dotted line corresponds to the PAAS (Post Archean Australian Shale; Taylor and McLennan, 1985). Normalizing values are from Nakamura (1974)

\subsubsection{Upper sequence}

The metapelitic schists of this sequence show less silicic $\left(\mathrm{SiO}_{2}=57.63-59.77\right)$ and more aluminic $\left(\mathrm{Al}_{2} \mathrm{O}_{3}=14.58-19.82 \mathrm{wt} . \%\right)$ compositions than the derital rocks from the lower sequence. They also have higher contents in $\mathrm{K}_{2} \mathrm{O}, \mathrm{Fe}_{2} \mathrm{O}_{3}, \mathrm{MgO}, \mathrm{MnO}$ and $\mathrm{TiO}_{2}$, and lower values in $\mathrm{Na}_{2} \mathrm{O}$ and $\mathrm{CaO}$ (Table 1). The $\mathrm{SiO}_{2} / \mathrm{Al}_{2} \mathrm{O}_{3}$ (2.9-3.1) and $\mathrm{Al}_{2} \mathrm{O}_{3}, \mathrm{TiO}_{2}$ (21.1-26.2) ratios fit those of the upper continental crust (McLennan,
2001). The schists are classified as shales in the diagram of Herron (1988). Sample B-21 is an arkose, but this is a local and very restricted composition in the upper sequence (Fig. 3a), and it is shown in Fig. $2 \mathrm{~b}$.

The total REE content ( $\Sigma R E E$ ) ranges between 88 and $235 \mathrm{ppm}$, with moderately to highly (sample B-21) fractionated chondrite-normalized patterns (Fig. 3C). The schist samples B-17, B-18 and B-19 show REE patterns almost identical to those of the greywackes of the lower sequence, in turn similar to the average composition of the upper continental crust represented by the PAAS (Taylor and McLennan, 1985). All the samples show a slight to pronounced $\mathrm{Eu}$ anomaly $\left(\mathrm{Eu} / \mathrm{Eu}^{*}=0.71-0.20\right)$. The samples with shale composition have an average $\mathrm{Th} / \mathrm{Sc}$ value of 0.7 , slightly lower than PAAS and probably suggesting the presence of an important volume of mafic rocks in the source area. This conclusion is also based on the $\mathrm{Ti} / \mathrm{Zr}$ (39.3; PAAS $=28.2$ ) and $\mathrm{Zr} / \mathrm{Sc}$ (6.6; PAAS $=13.1)$ ratios, as well as on the high $V$ contents (124-164 ppm).

\subsection{Tectonic setting}

The tectonic discrimination diagrams developed by Bathia and Crook (1986) allow to distinguish clearly among the four tectonic settings considered to be the most common environments for greywacke deposition: (A) oceanic island arc, (B) continental island arc, (C) active continental margin, and (D) passive margin. Only siliciclastic rocks from the lower sequence showing greywacke compositions were plotted in these diagrams (Fig. 4). The schists from the upper sequence do not meet the requirements (pelitic composition) to be plotted in them, and were consequently ruled out for tectonic discrimination studies.

The diagrams presented in Fig. 4 allow to discard the type-A setting for the sedimentation of these greywackes, as their origin seems clearly related to a tectonic setting with the presence of an older continental crust. In the Th-Co-Zr diagram the metagreywackes generally plot in the B field, which points to a basin located either in an island arc built on a mature continental crust, or in a magmatic arc built on a thinned continental margin. On the other hand, the Th-Sc$\mathrm{Zr}$ and Ia-Th-Sc diagrams suggest some passive margin affinity. This affinity is especially clear in Fig. $4 \mathrm{~b}$, but can be also deduced from the diagram $4 \mathrm{c}$ where it is not possible to differentiate type- $\mathrm{C}$ or type-D greywackes, although the incompatibility with type- $C$ is clear after projections in Fig. $4 \mathrm{a}$ and b. In summary and according to the diagrams of Bathia and Crook (1986), the metagreywackes from the lower sequence were generated in relation to a magmatic arc built on a thinned continental margin, but probably closer to the main continental domain and to certain distance from the areas with the most important magmatic activity. This setting would probably explain the observed relative affinity to passive margins. A backarc setting or a retro-arc setting would explain the compositions observed in the metagreywackes, as well as the whole sedimentary characteristics of the lower sequence. This tectonic setting was previously suggested by Díez Fernández et al. (2010) and it will be examined in more detail in a next section.

Fig. 5 shows PAAS-normalized plots of the most significant elements for tectonic setting discrimination. The diagrams are plotted according to the criteria of Thompson (1982). The patterns defined by the metagreywackes (Fig. Sa) are quite similar to those typical of continental island arc or active margins (Winchester and Max, 1989). The plots are characterized by depletion in most of the large ion lithophile elements (LLE: $\mathrm{Cs}, \mathrm{Rb}, \mathrm{Th}, \mathrm{Ce}$ and $\mathrm{K}_{2} \mathrm{O}$ ), which deviate slightly from 1 , with the exception of Ia, $\mathrm{U}$ and $\mathrm{P}_{2} \mathrm{O}_{5}$. The high field strength elements (HFSE: $\mathrm{Zr}, \mathrm{Hf}, \mathrm{HREE}, \mathrm{Sm}, \mathrm{TiO}_{2}$ and $\mathrm{Sc}$ ) are generally close to 1 , with a slight characteristic negative $\mathrm{TiO}_{2}$ anomaly, typical of this type of greywackes (Winchester and Max, 1989). In general a flat pattern is observed, with an average close or below 1 . It can be also stressed the general absence of a pronounced $\mathrm{Sr}$ anomaly, typical of the most characteristic passive margins. The schists of the upper sequence show more variable patterns, specially when the anomalous 

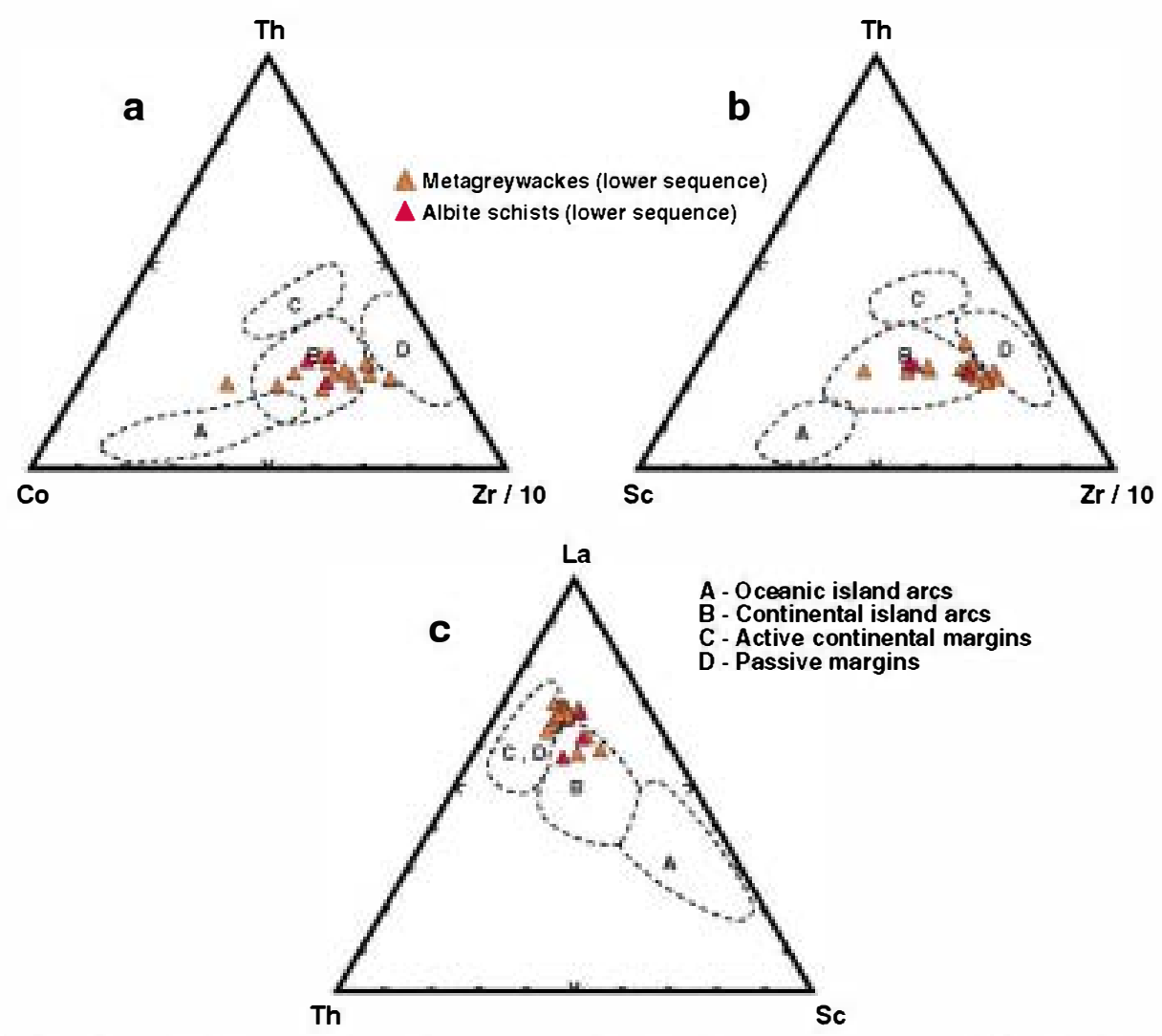

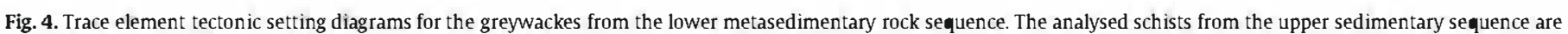
not included in these diagrams, as they show a scattered projection due to compositions different from typical greywackes. Diagrams are after Bathia and Crook (1986).

sample B-21 (arkose) is considered. However, samples B-17, B-18 and B-19 show patterns close to 1 with some differences in relation to the metagreywackes from the lower sequence. They do not show depletion in some LILE elements ( $\mathrm{Rb}, \mathrm{Th}, \mathrm{Ce}, \mathrm{K}_{2} \mathrm{O}$ ) although negative anomalies exist for $\mathrm{U}, \mathrm{Sr}, \mathrm{Hf}$ and $\mathrm{TiO}_{2}$, which could suggest passive margin affinity (Fig. 5b).

\subsection{Sm-Nd isotope systematics}

The Sm-Nd isotopic analyses were performed at the Centro de Geocronología y Geoquímica Isotópica from the Universidad Complutense de Madrid. They were carried out in whole-rock powders using a ${ }^{150} \mathrm{Nd}-{ }^{149} \mathrm{Sm}$ tracer by isotope dilution-thermal ionization mass spectrometry (ID-TIMS). The samples were first dissolved through oven digestion in sealed Teflon bombs with ultra pure reagents to perform two-stage conventional cation-exchange chromatography for separation of Sm and Nd (Strelow, 1960; Winchester, 1963), and subsequently analysed using a Sector 54 VG-Micromass multicollector spectrometer. The measured ${ }^{143} \mathrm{Nd} /{ }^{144} \mathrm{Nd}$ isotopic ratios were corrected for possible isobaric interferences from ${ }^{142} \mathrm{Ce}$ and ${ }^{144} \mathrm{Sm}$ (only for samples with ${ }^{147} \mathrm{Sm} /{ }^{144} \mathrm{Sm}<0.0001$ ) and normalized to ${ }^{146} \mathrm{Nd} /{ }^{144} \mathrm{Nd}=0.7219$ to correct for mass fractionation (Table 3 ). The Lajolla Nd international isotopic standard was analysed during sample measurement, and gave an average value of ${ }^{143} \mathrm{Nd} /{ }^{144} \mathrm{Nd}=0.5114840$ for 9 replicas, with an internal precision of $\pm 0.000032(2 \sigma)$. These values were used to correct the measured ratios for possible sample drift. The estimated error for the ${ }^{147} \mathrm{Sm} /{ }^{144} \mathrm{Nd}$ ratio is $0.1 \%$.

In crustal evolution models based on $\mathrm{Nd}$ isotopic composition, the main fractionation event during the formation and evolution of continental crust takes place during partial melting of lithospheric mantle to generate crustal rocks (McLennan and Hemming, 1992). The $\varepsilon N d$ model age of a sedimentary rock represents the average age of the extraction of its components from the mantle. In the case of detrital rocks, model ages usually reflect complex mixing based on the different age and provenance of their components. The combined interpretation of $\mathrm{Nd}$ model ages and detrital zircon ages has proven to be a powerful tool for investigating the evolution of continental crust, especially in orogenic belts (e.g., linnemann et al., 2004). The investigated metasedimentary rocks show relatively uniform $\varepsilon \mathrm{Nd}(0)$ values, ranging between -19.0 and -12.3 (average -16.4 ; Table 3 ). $\varepsilon \mathrm{Nd}(\mathrm{T})$ ranges -13.1 to -8.1 in the lower sequence (maximum depositional age of $560 \mathrm{Ma}$; Díez Fernández et al, 2010) and -10.1 to -8.5 in the upper sequence (maximum depositional age of $500 \mathrm{Ma}$; Diez Fernández et al., 2010). The analysed metasedimentary rocks show ${ }^{147} \mathrm{Sm} /{ }^{144} \mathrm{Sm}$ ratios ranging between 0.1055 and 0.1464 , with average at 0.1174 . This is a ratio below the upper limit of ${ }^{147} \mathrm{Sm} /{ }^{144} \mathrm{Sm}=0.165$ suggested by Stern (2002) appropriate to perform $\mathrm{NdT}_{\mathrm{DM}}$ calculations. The $\mathrm{T}_{\mathrm{DM}}$ model ages (DePaolo, 1981) are Paleoproterozoic and range between 2223 and $1782 \mathrm{Ma}$, with an average value of $1919 \mathrm{Ma}$ (Fig. 6). A collection of $\mathrm{Nd}$ model ages from different regions (Linnemann and Romer, 2002) is also included in Fig. 6. These ages have been divided into two groups according to the age of the dominant source (Grenvillian and post-Grenvillian/pre-Cadomian crust, or preGrenvillian, >0.9-1.1 Ga. cratonic crust). In this collection of $\mathrm{Nd}$ model ages, similar Paleoproterozoic ages are only reported in elements of the West African Craton, the Amazonian Craton and the Icartian gneisses of Brittany. The rest of terranes included in this collection show younger Nd model ages. Moreover, Fig. 6 also includes for comparison Nd data of the uppermost greywackes of the Órdenes Complex (upper units; Fuenlabrada et al., 2010). These Cambrian greywackes (maximum age of sedimentation at 530-500 Ma) have much younger model ages ranging between 1215 and $720 \mathrm{Ma}$. 


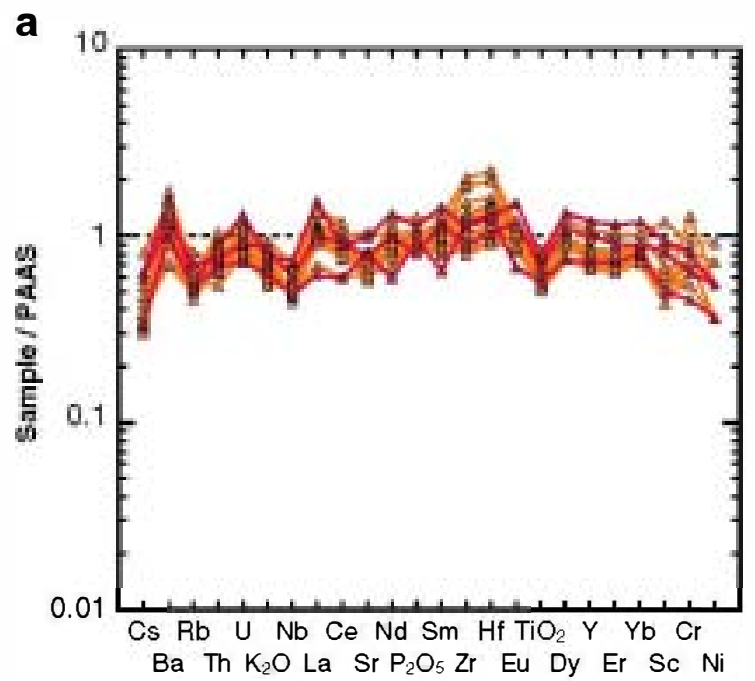

b

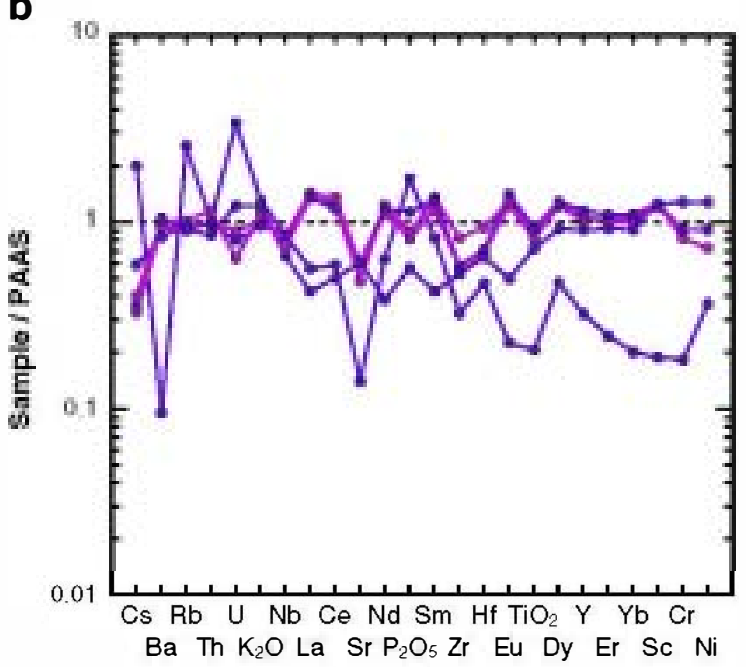

arc/retro-arc setting, closer to the external platform of the Gondwana Mainland, in a region where the most important sedimentary supplies would come from source areas located in the interior of the continental domain. The influence of the Cadomian magmatic arc on the composition of the greywackes is clear, as indicated by their detrital zircon age populations (Díez Fernández et al., 2010). However, the sedimentary basin was apparently located far away from the region with the most important igneous activity, because the oldest igneous rocks intruding the greywackic sequence are Middle Cambrian.

The origin of the Cambrian sedimentary rocks of the upper sequence is more uncertain, as they are mainly constituted by metapelitic schists whose compositions are not ideal for tectonic setting discrimination. The available geochemical data are not abundant, but they seem to suggest some affinity with passive margin sediments; i.e., these sediments were probably deposited close to the continental domain and to certain distance from the most active zones in the magmatic arc. The absence of intrusive granitic rocks, which are widespread in the lower sequence, supports this interpretation (Fig. 2b). It is likely that during Late Cambrian times the previously deposited series of the lower sequence were located towards the most active part of the arc system, while the sediments of the upper sequence were deposited closer to the continental platform. In addition, it is important to consider that the contact between upper and lower metasedimentary sequences is a low-angle fault (extensional detachment), so these series could have been located at different positions along the margin. On the other hand, opening of the Rheic Ocean and transition of the Gondwana margin to a typical passive margin setting occurred between the Middle Cambrian and the Early Ordovician (Arenas et al., 2007; Murphy et al., 2010; Nance et al., 2010). This agrees with the observed sedimentary evolution and explains the presence of N-MORB mafic rocks in the sedimentary series (Rodríguez Aller, 2005), which are particularly more abundant in the upper sequence (Fig. 2b). Although there are not many geochemical data about the metasedimentary rocks of the upper sequence, the compositional patterns (PAAS-normalized plots) of some samples (Fig. Sb) could indicate an evolution from a back-arc setting to a passive margin. This transition might be related either with the

Table 3

Whole rock Nd isotope data of metasedimentary rocks.

\begin{tabular}{|c|c|c|c|c|c|c|c|c|}
\hline Sample & $\mathrm{Sm}$ & $\mathrm{Nd}$ & $\begin{array}{l}{ }^{147} \mathrm{Sm} / \\
{ }^{144} \mathrm{Nd}\end{array}$ & $\begin{array}{l}{ }^{143} \mathrm{Nd} / \\
{ }^{144} \mathrm{Nd}\end{array}$ & $2 \sigma$ & $\varepsilon \mathrm{Nd}(0)$ & $\varepsilon N d(560)^{a}$ & $\begin{array}{l}\text { IDM } \\
(\mathrm{Ma})^{\mathrm{b}}\end{array}$ \\
\hline B- 1 & 4.00 & 20.67 & 0.1169 & 0.511676 & 3 & -18.8 & -13.1 & 2078 \\
\hline B-2 & 4.14 & 21.83 & 0.1146 & 0.511670 & 3 & -18.9 & -13.0 & 2041 \\
\hline B-3 & 4.75 & 23.42 & 0.1227 & 0.511950 & 3 & -13.4 & -8.1 & 1782 \\
\hline B-4 & 4.10 & 21.31 & 0.1164 & 0.511852 & 3 & -15.3 & -9.6 & 1817 \\
\hline B-5 & 4.49 & 22.80 & 0.1191 & 0.511800 & 4 & -16.3 & -10.8 & 1940 \\
\hline B- 6 & 3.73 & 19.69 & 0.1146 & 0.511731 & 3 & -17.7 & -11.8 & 1956 \\
\hline B-7 & 3.03 & 14.81 & 0.1239 & 0.511809 & 4 & -16.2 & -11.0 & 2020 \\
\hline B- 8 & 5.58 & 30.14 & 0.1118 & 0.511712 & 3 & -18.1 & -12.0 & 1934 \\
\hline B-9 & 5.62 & 29.26 & 0.1162 & 0.511844 & 4 & -15.5 & -9.7 & 1824 \\
\hline B- 10 & 3.33 & 17.93 & 0.1123 & 0.511727 & 6 & -17.8 & -11.7 & 1921 \\
\hline B-11 & 6.24 & 35.72 & 0.1055 & 0.511663 & 3 & -19.0 & -12.5 & 1893 \\
\hline B- 12 & 4.41 & 22.83 & 0.1167 & 0.511728 & 3 & -17.7 & -12.0 & 1999 \\
\hline B- 13 & 3.61 & 19.94 & 0.1095 & 0.511708 & 3 & -18.1 & -11.9 & 1898 \\
\hline B- 14 & 4.05 & 21.25 & 0.1152 & 0.511729 & 3 & -17.7 & -11.9 & 1971 \\
\hline B- 15 & 3.71 & 19.80 & 0.1133 & 0.511753 & 3 & -17.3 & -11.3 & 1902 \\
\hline B- 16 & 4.04 & 21.90 & 0.1115 & 0.511700 & 3 & -18.3 & -12.2 & 1946 \\
\hline B-22 & 2.46 & 12.91 & 0.1150 & 0.511771 & 3 & -16.9 & -11.1 & 1906 \\
\hline B-23 & 4.55 & 22.19 & 0.1238 & 0.511885 & 3 & -14.7 & -9.5 & 1902 \\
\hline B- 17 & 4.84 & 26.06 & 0.1123 & 0.511846 & 4 & -15.4 & -10.1 & 1756 \\
\hline B- 18 & 5.19 & 26.38 & 0.1189 & 0.511877 & 3 & -14.9 & -9.9 & 1824 \\
\hline B-19 & 5.33 & 26.66 & 0.1208 & 0.511954 & 4 & -13.3 & -8.5 & 1743 \\
\hline B-20 & 1.87 & 9.26 & 0.1223 & 0.511892 & 3 & -14.6 & -9.8 & 1863 \\
\hline B-21 & 3.05 & 12.60 & 0.1464 & 0.512007 & 3 & -12.3 & $-\mathbf{9} .1$ & 2223 \\
\hline
\end{tabular}

a $\varepsilon N d(t)$ calculated for $560 \mathrm{Ma}$.

b Nd model ages calculated according to DePaolo (1981).
Fig. 5. (a, b) PAAS-normalized trace elements plots for the metasedimentary rocks from the lower and upper sequences, respectively. PAAS after Taylor and McLennan (1985).

\section{Discussion}

Different paleogeographic models have shown that large magmatic arcs developed in the peri-Gondwanan realm. Their activity took place between Neoproterozoic (Cadomian-Pan-African cycle) and Early Ordovician times (Murphy and Nance, 2002; Stampfli and Borel, 2002), and they left an imprint in the sedimentological and magmatic record of many of the terranes forming part of the Variscan Belt (Linnemann et al., 2004, 2007; Rodríguez Alonso et al., 2004; Ugidos et al., 2003). The geochemical features of the metagreywackes of the lower sequence suggest that they were generated in relation to an arc system built on a thinned continental margin, although they also record certain chemical transition to passive margin greywackes. A back-arc setting or a retro-arc setting as those presented in the models of Fig. 7 (a, b), would explain most of the geochemical features observed in these lithologies. The lower sequence of the basal units was probably deposited in an Ediacaran-Early Cambrian back- 


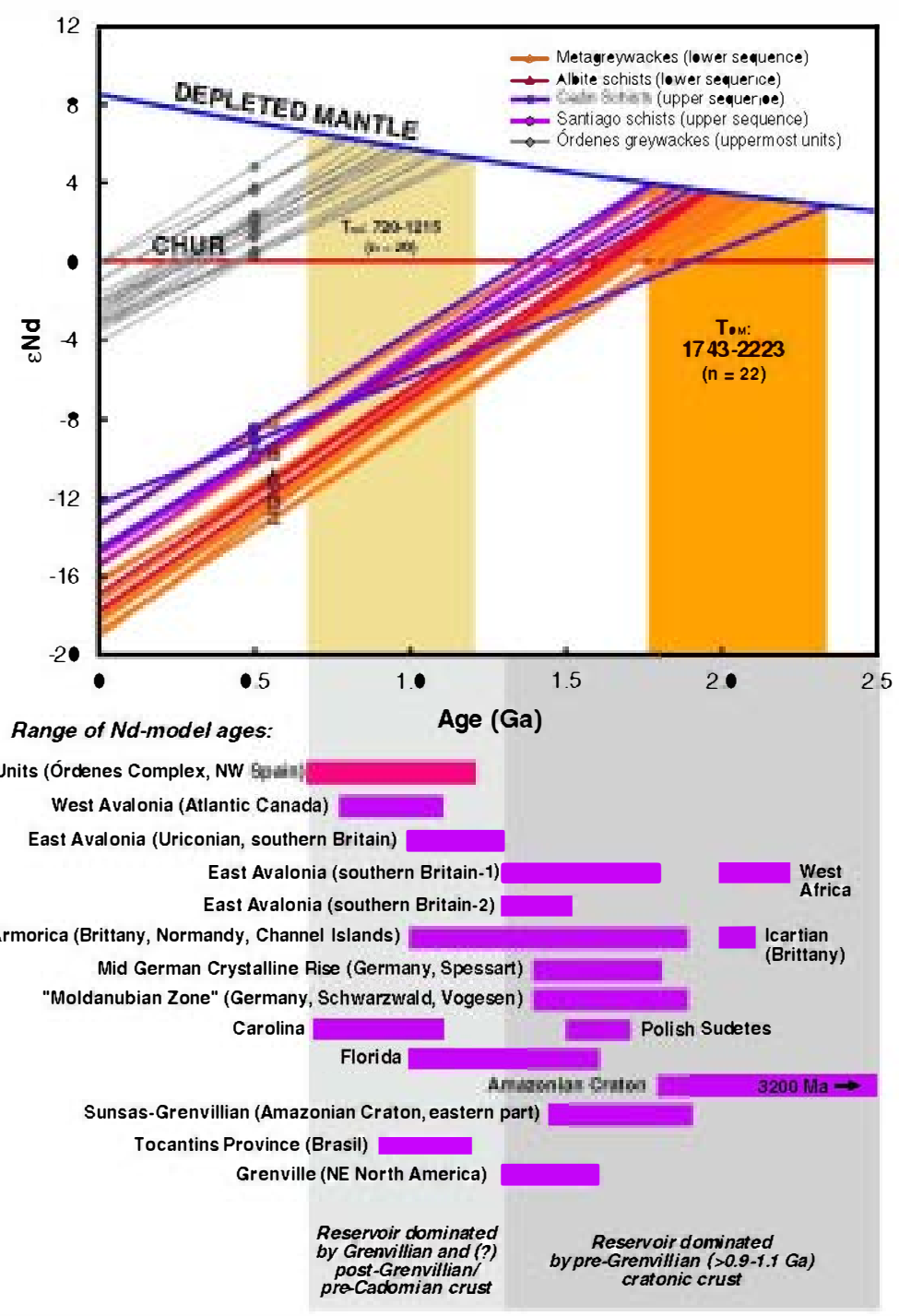

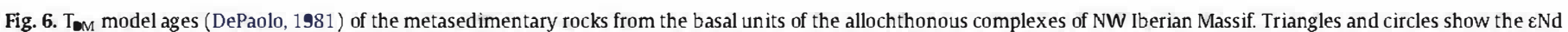

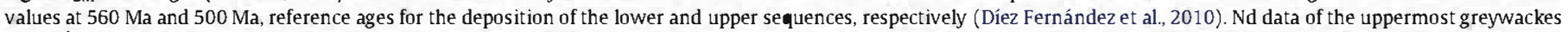

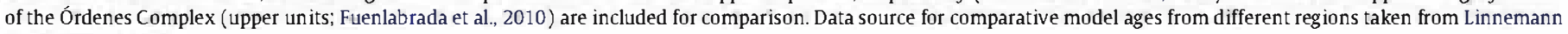
and Romer (2002).

attenuation of the magmatic activity in the arc, or the progressive ocean ward translation of this activity, or the drifting of the whole arc system away from the continental margin.

The Nd model ages of the 23 analysed samples are Paleoproterozoic and range between $1782 \mathrm{Ma}$ and $2223 \mathrm{Ma}$ (average value 1919; Fig. 6). The Nd model ages are slightly younger in the upper sequence than in the lower sequence, but altogether they define a single population. Therefore the two metasedimentary rock sequences can be clearly related. Sedimentation probably took place in the same basin located in the continental platform of Gondwana, whose main source areas did not change during the Late Neoproterozoic and Cambrian times. The $\mathrm{Nd}$ model ages are very old and they seem to be only compatible with Paleoproterozoic-Archean source areas. The participation of younger sources was probably limited, although both the metagreywackes and the metapelitic rocks contain Mesoproterozoic and Neoproterozoic (actually the most abundant grains) detrital zircon populations (Diez Fernández et al, 2010). The chronology recorded by the main detrital zircon population and by the $\mathrm{NdT}_{\mathrm{DM}}$ is not contradictory as each method supplies a different information (Liégeois and Stern, 2010). Source areas dominated by Paleoproterozoic-Archean materials intruded by Pan-African granitoids, and with variable participation of detrital sedimentary rock series through time, explain the Sm-Nd isotopic data and the age populations of detrital zircons (see Liégeois and Stern, 2010, and references therein). The significant difference between the ages reported by the two methods suggests that the involved PanAfrican granitoids were the result of partial melting of Paleoproterozoic-Archean basement and its erosion products, and consequently they share a same $\mathrm{Sm}-\mathrm{Nd}$ isotope signature. The important participation of Neoproterozoic zircons can be explained by the high abundance of zircons in the Cadomian-Pan-African granitoids (750-550 Ma), as it is known that granitoids are the most important source for detrital zircons. The North African margin of Gondwana shows suitable features to be considered the source area for these sedimentary rocks. It is difficult to ascertain the precise location of the sedimentary basin, because its position can only be determined presently using provenance studies based in U-Pb geochronology of dewital zircons. In the context of North Africa a key element is the presence/absence of a Stenian-Tonian population of detrital zircons (750-1150 Ma), which it is also present in the sedimentary series of the basal units. Avigad et al. (2012) suggested that Gondwanan series 

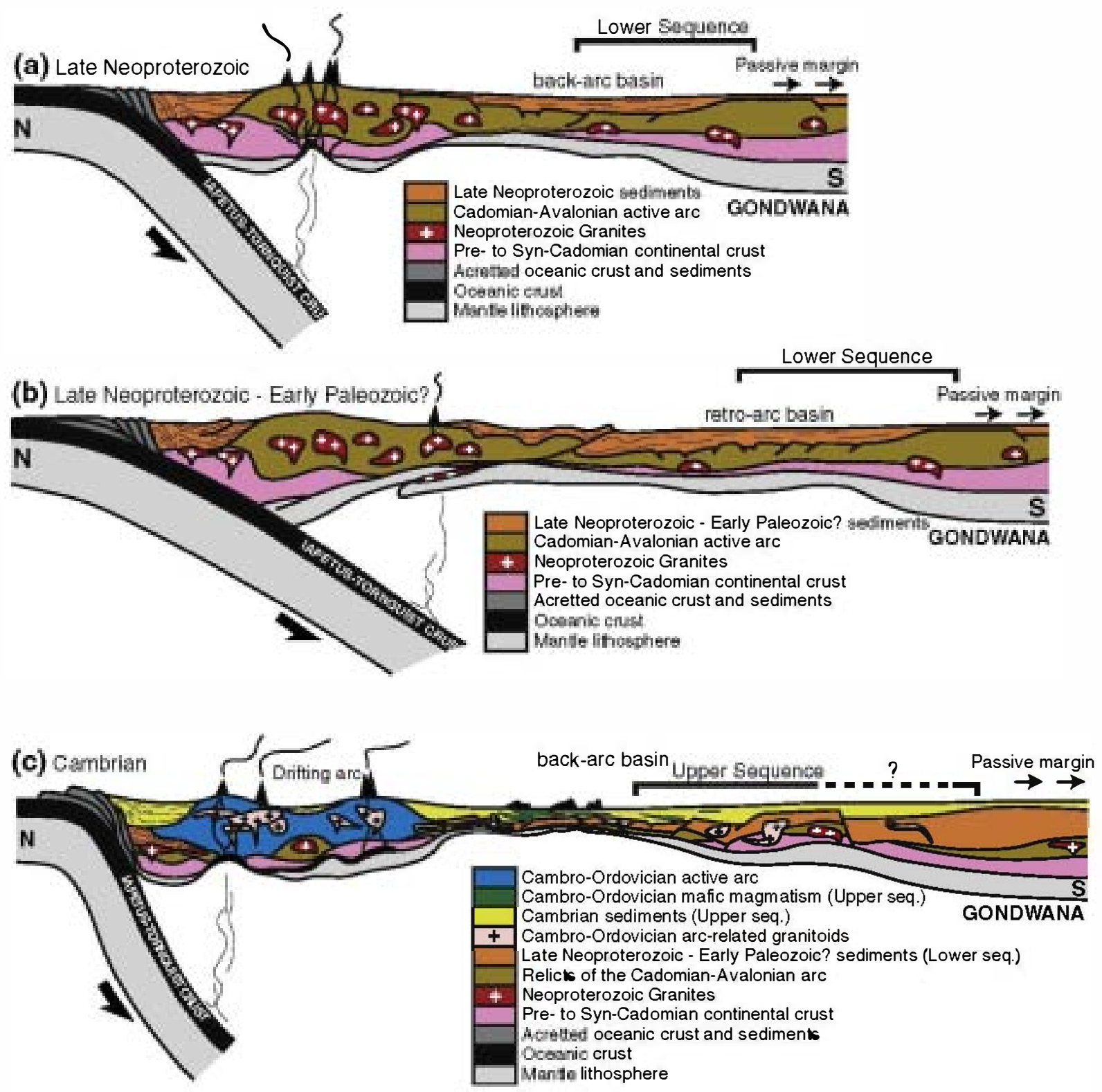

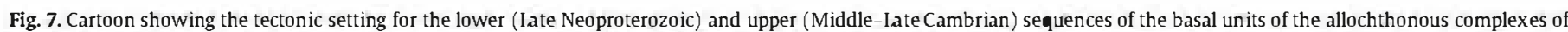

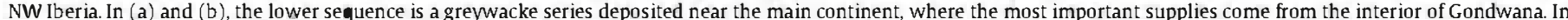

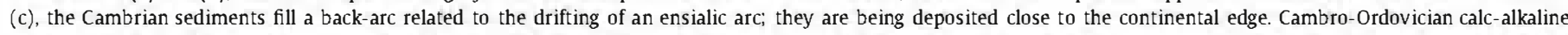

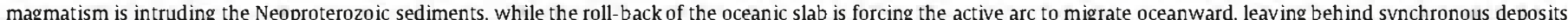

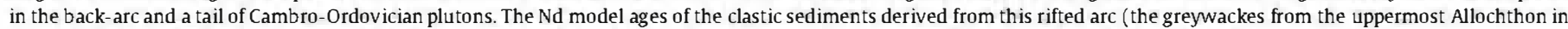
Fig. 6) indicates contribution from juvenile sources. These models refine previous interpretations presented by Díez Fernández et al. (2010).

from Morocco include these zircons only since Middle Cambrian times, without any significant presence before this period (see also Ábalos et al., 2012; Abati et al., 2010a; Boher et al., 1992; Potrel et al., 1998). The presence of Stenian-Tonian detrital zircons is more common in the Saharan Craton, also in Lower Cambrian-Neoproterozoic sedimentary series (Avigad et al., 2003, 2012). Anyway the existence of large outcrops of primary Stenian-Tonian materials is unknown in North Africa, and thence the actual source of these zircons is enigmatic suggesting long transport from source areas located far away. Using this information, Díez Fernández et al. (2010) suggested that sediments from the basal units were deposited in basins probably located in an intermediate position between the West African Craton and the Saharan Craton. This interpretation is very sensitive to the actual age of the studied series, to which only a maximum depositional age approach based in detrital zircons is available, but it is coherent with a provenance not toofar from the present location of the investigated allochthonous sedimentary rock series.

The Nd model ages of the sedimentary sequences of the basal units are considerably older than those of the Cambrian greywackes (530-500 Ma) of the upper units of the same region, which range between 720 Ma and $1215 \mathrm{Ma}$ (Fuenlabrada et al., 2010; Fig. 6). Separated from the basal units by ophiolites, the upper units have been repeatedly interpreted as a well-preserved section of a periGondwanan arc (Abati et al., 2003, 2007; Díaz García et al., 2010; Fuenlabrada et al., 2010), what suggests that these relatively young $\mathrm{Nd}$ model ages are influenced by abundant supplies of juvenile material generated in relation to the magmatic activity of the arc (see Fig. 7c). 
Comparison of the Sm-Nd geochemistry of metasedimentary rocks from terranes located in the footwall and hanging wall to the Variscan suture, may help to reconstruct the paleogeography of the peri-Gondwanan domain during Ediacaran-Early Paleozoic times. This information can also be valuable in order to recognize and correlate the allochthonous terranes located to both sides of the suture zone in central and Western Europe, especially in regions where the position of the suture is not clear or the structural relationships are under discussion.

\section{Acknowledgments}

Financial support for this research has been provided by the Spanish project CGI2007-65338-CO2/BTE (Ministerio de Ciencia e Innovación). Insightful reviews of the manuscript performed by Ulf Linnemann and an anonymous referee are gratefully acknowledged, as well as the important editorial work of Marco Scambelluri that significantly improved the final version of the manuscript.

\section{References}

Ábalos, B., Gil Ibarguchi, J.I., Sánchez-Lorda, M.E., Paquette, J.L., 2012. African/Amazonian Proterozoic correlations of Iberia: A detrital ziroon U-Pb study of early Cambrian conglomerates from the Sierra de la Demanda (northern Spain). Tectonics 31, TC3003.

Abati.J., Dunning, G.R., Arenas, R., Díaz García, F., González Cuadra, P., Martínez Catalán, J.R., Andonaegui, P., 1999. Early Ordovician orogenic event in Galicia (NW Spain): evidences from $\mathrm{U}-\mathrm{Pb}$ ages in the uppermost unit of the Órdenes Complex. Earth and Planetary Science Letters $165,213-228$.

Abati,J., Arenas, R., Martínez Catalán, J.R., Díaz García, F., 2003. Anticlockwise P-T path of granulites from the Monte Castelo Gabbro (Órdenes Complex, NW Spain). Journal of Petrology 44, 305-327.

A bati, J., Castiñeiras, P., Arenas, R., Fernández-Suárez, J., Gómez Barreiro, J., Wooden, J. 2007. Using SHRIMP-RG U-Pb zircon dating to unravel tectonomagmatic events in arc environments. A new peri-Gondwanan terrane in Iberia? Terra Nova 19 432-439.

Abati, J., Aghzer, A.M., Gerdes, A., Ennih, N., 2010a. Detrital zircon ages of Neoproterozoic sequences of the Moroccan Anti-Atlas belt. Precambrian Research $181,115-128$.

Abati, J., Gerdes, A., Fernández-Suárez, J., Arenas, R., Whitehouse, M.J., Díez Fernández, R., 2010b. Magmatism and early-Variscan continental subduction in the northern Gondwana margin recorded in zircons from the basal units of Galicia, NW Spain. Geological Society of America Bulletin 122, 219-235.

Arenas, R., Gil Ibarguchi,J.I., González Lodeiro, F., Klein, E., Martínez Catalán, J.R., Ortega Gironés, E., de Pablo Maciá, J.G., Peinado, M., 1986. Tectonostratigraphic units in the complexes with mafic and related rocks of the Iberian Massif. Hercynica 2 $87-110$.

Arenas, R., Martínez Catalán, J.R., Sánchez Martínez, S., Fernández-Suárez, J. Andonaegui, P., Pearce, J.A. Corfu, F. 2007. The Vila de Cruces Ophiolite: A remnan of the Early Rheic Ocean in the Variscan suture of Galicia (Northwest Iberian Massif). Journal of Geology 115, 129-148.

Arenas, R., Sánchez Martínez, S., Castiñeiras, P. Jeffries, T.E., Díez Fernández, R Andonaegui, P., 2009. The basal tectonic mélange of the Cabo Ortegal Complex (NW Iberian Massif): a key unit in the suture of Pangea. Journal of Iberian Geology $35,85-125$.

Avigad, D., Kolodner, K., McWilliams, M., Persing, H., Weissbrod, T., 2003. Origin of northern Gondwana Cambrian sandstone revealed by detrital zircon SHRIMP dating. Geology 31, 227-230

Avigad, D., Gerdes, A., Morag, N., Bechstädt, T., 2012. Coupled U-Pb-Hf of detrital zir cons of Cambrian sandstones from Morocco and Sardinia: Implications for provenance and Precambrian crustal evolution of North Africa. Gondwana Research 21 $690-703$.

Bathia, M.R., 1983. Plate tectonics and geochemical composition of sandstones. Journal of Geology 91, 611-627.

Bathia, M.R., Crook, K.A.W., 1986. Trace elements characteristics of greywackes and tectonic setting discrimination of sedimentary basins. Contributions to Mineralogy and Petrology 92, 181-193.

Boher, M., Abouchami, W., Michard, A., Albarede, F., Arndt, N.T., 1992. Crustal growth in West Africa at 2.1 Ga. Journal of Geophysical Research 97, 345-369.

Crook, K.A.W., 1974. Lithogenesis and geotectonics: the significance of compositional variations in flysch arenites (greywackes). In: Doti, R.H., Shaver, R.H. (Eds.), Modern and ancient geosynclinal sedimentation Society of Economic Palaeontologists and Mineralogists Special Publication 19, 304-310.

DePaolo, D.J., 1981. Neodymium isotopes in the Colorado Front Range and crust-mantle evolution in the Proterozoic. Nature 291, 193-196.

Díaz García, F., Arenas, R., Martínez Catalán, J.R., González del Tánago, J., Dunning, G.R., 1999. Tectonic evolution of the Careón Ophiolite (northwest Spain): a remnant of oceanic lithosphere in the Variscan Belt. Journal of Geology 107, 587-605.
Díaz García, F., Sánchez Martínez, S., Castiñeiras, P., Fuenlabrada, J.M., Arenas, R., 2010. A peri-Gondwanan arc in NW Iberia. II: Assessment of the intra-arc tectonothermal evolution through U-Pb SHRIMP dating of mafic dykes. Gondwana Research 17, $352-362$.

Díez Fernández, R., 2011. Evolución estructural y cinemática de una corteza continental subducida: la unidad de Malpica-Tui (NO del Macizo Ibérico). Nova Terra $40228 \mathrm{pp}$.

Díez Fernández, R. Martínez Catalán, J.R., Gerdes, A., Abati, J., Arenas, R., FernándezSuárez, J., 2010. U-Pb ages of detrital zircons from the basal allochthonous units of NW Iberia: Provenance and paleoposition on the northern margin of Gondwana during the Neoproterozoic and Paleozoic. Gondwana Research 18, 385-399.

Díez Fernández, R., Martínez Catalán, J.R., Arenas, R., Abati, J., 2011. Tectonic evolution of a continental subduction-exhumation channel: Variscan structure of the basal allochthonous units in NW Spain. Tectonics 30, TC3009.

Díez Fernández, R., Castiñeiras, P., Gómez Barreiro, J., 2012. Age constraints on Lower Paleozoic convection system: magmatic events in the NW Iberian Gondwana margin. Gondwana Research 21, 1066-1079.

Drost, K., Linnemann, U., McNaughton, N., Fatka, O., Kraft, P., Gehmlich, M., Tonk, C. Marek, J., 2004. New data on the Neoproterozoic-Cambrian geotectonic setting of the Teplá-Barrandian volcano-sedimentary succesions: geochemistry, U-Pb zircon ages, and provenance (Bohemian Massif, Czech Republic). International Journal of Earth Sciences 93, 742-757.

Fernández-Suarez, J., Corfu, F., Arenas, R., Marcos, A., Martínez Catalán, J.R., Díaz García, F., Abati, J., Fernández, F.J., 2002. U-Pb evidence for a polymetamorphic evolution of the HP-HT units of the NW Iberia Massif. Contributions to Mineralogy and Petrology 143, 236-253.

Fernández-Suárez, J., Arenas, R., Abati, J., Martínez Catalán, J.R., Whitehouse, M.J., Jeffries, T.E., 2007. U-Pb chronometry of polymetamorphic high-pressure granulites: An example from the allochthonous terranes of the NW Iberian Variscan belt. In: Hatcher Jr., R.D., Carlson, M.P., McBride, J.H., Martínez Catalán, J.R. (Eds.), 4-D Framework of continental crustGeological Society of America Memoir 200, 469-488.

Fuenlabrada, J.M., Arenas, R., Sánchez Martínez, S., Díaz García, F., Castiñeiras, P., 2010. A peri-Gondwanan arc in NW Iberia. I: Isotopic and geochemical constraints on the origin of the arc-A sedimentary approach. Gondwana Research 17, 338-351.

Gil Ibarguchi, J.I., Ortega Gironés, E., 1985. Petrology, structure and geotectonic implications of glaucophane-bearing eclogites and related rocks from the Malpica-Tuy (MT) unit, Galicia, Nortwest Spain. Chemical Geology 50, 145-162

Gómez Barreiro, J., Martínez Catalán,J.R., A renas, R., Castiñeiras, P., Abati, J., Díaz García, F., Wijbrans, J.R., 2007. Tectonic evolution of the upper allochthon of the Órdenes complex (northwestern Iberian Massif): Structural constraints to a polyorogenic peri-Gondwanan terrane. In: Linneman, U., Nance, R.D., Kraft, P., Zulauf, G. (Eds.), The evolution of the Rheic Ocean: From Avalonian-Cadomian active margin to Alleghenian-Variscan collisionGeological Society of America Special Paper 423, 315-332.

Gómez Barreiro, J., Martínez Catalán, J.R., Prior, D., Wenk, H.-R., Vogel, S., Díaz García, F., Arenas, R., Sánchez Martínez, S., Lonardelli, I., 2010. Fabric development in a Middle Devonian intra-oceanic subduction regime: the Careón ophiolite (NW Spain). Journal of Geology 118, 163-186.

Hatcher Jr. R. D. Bream, B. R., Merschat, A.J. 2007. Tectonic map of the southern and central Appalachians: A tale of three orogens and a complete Wilson cycle. In: Hatcher Jr., R.D., Carlson, M.P., McBride, J.H., Martínez Catalán, J.R. (Eds.), 4-D Framework of continental crustGeological Society of America Memoir 200, $595-632$.

Hegde, V.S., Chavadi, V.C., 2009. Geochemistry of Late Archaean metagreywackes from the Western Dharwar Craton, South India: Implications for provenance and nature of the Iate Archaean crust. Gondwana Research 15, 178-187.

Herron, M.M., 1988. Geochemical classification of terrigenous sands and shales from core or log data. Journal of Sedimentary Petrology 58, 820-829.

Liégeois, J.P., Stern, R.J., 2010. Sr-Nd isotopes and geochemistry of granite-gneiss complexes from the Meatiq and Hafafit domes, Eastern Desert, Egypt: No evidence for pre-Neoproterozoic crust. Gondwana Research 57, 31-40.

Linneman, U. McNaughton, N.J. Romer, R.L. Gehmlich, M. Drost, K. Tonk C, 2004. West African provenance for Saxo-Thuringia (Bohemian Massif): Did Armorica ever leave pre-Pangean Gondwana?-U/Pb-SHRIMP zircon evidence and the Ndisotopic record. International Journal of Earth Sciences 93, 683-705.

Linnemann, U., Romer, R.L, 2002. The Cadomian Orogeny in Saxo-Thuringia, Germany: geochemical and $\mathrm{Nd}-\mathrm{Sr}-\mathrm{Pb}$ isotopic characterization of marginal basins with constraints to geotectonic setting and provenance. Tectonophysics $352,33-64$

Linnemann, U., Gerdes, A., Drost, K., Buschmann, B., 2007. The continuum between Cadomian orogenesis and opening of the Rheic Ocean: Constraints from IA-ICP-MS $\mathrm{U}-\mathrm{Pb}$ ziroon dating and analysis of plate-tectonic setting (Saxo-Thuringian zone, northeastern Bohemian Massif, Germany). In: Linnemann, U. Nance, RD, Kraft, P. Zulauf, G. (Eds.), The evolution of the Rheic Ocean: From Avalonian-Cadomian active margin to Alleghenian-Variscan collisionGeological Society of America Special Paper 423, 61-96.

Martínez Catalán, J.R., Arenas, R., Díaz García, F., Rubio Pascual, F.J., Abati, J., Marquínez, J., 1996. Variscan exhumation of a subducted Paleozoic continental margin: The basal units of the Órdenes Complex, Galicia, NW Spain. Tectonics 15, 106-121.

Martínez Catalán, J.R., Arenas, R., Díaz García, F., González Cuadra, P., Gómez-Barreiro, J., Abati, J., Castiñeiras, P., Fernández-Suárez, J., Sánchez Martínez, S., Andonaegui, P., González Clavijo, E., Díez Montes, A., Rubio Pascual, F.J., Valle Aguado, B., 2007. Space and time in the tectonic evolution of the northwestern Iberian Massif: Implications for the Variscan belt. In: Hatcher Jr., R.D., Carlson, M.P., McBride, J.H. Martínez Catalán, J.R. (Eds.), 4-D Framework of continental crustGeological Society of America Memoir 200, 403-423. 
Martínez Catalán, J.R., Arenas, R., Abati, J., Sánchez Martínez, S., Díaz García, F., Fernández-Suárez, J., González Cuadra, P., Castiñeiras, P. Gómez Barreiro, J., Díez Montes, A., González Clavijo, E., Rubio Pascual, F.J. Andonaegui, P., Jeffr ies, T.E. Alcock, J.E., Díez Fernández, R., Iópez Carmona, A, 2009. A rootless suture and the loss of the roots of a mountain chain: The Variscan belt of NW Iberia. Comptes Rendus 341, 114-126.

McLennan, S.M., 2001. Relationships between the trace element composition of sedimentary rocks and upper continental crust. Geochemistry, Geophysics, Geosystems 2 art. no. 2000GC000109.

McLennan, S.M., Hemming, S.R., 1992. Samarium/neodymium elemental and isotopic systematics in sedimentary rocks. Geochimica et Cosmochimica Acta 56, 887-898.

McLennan, S.M., Hemming, S.R., McDaniel, D.K., Hanson, G.N., 1993. Geochemical approaches to sedimentation, provenance and tectonics. In: Johnssons, M.J., Basu, A. (Eds.), Processes controlling the composition of clastic sedimentsGeological Society of America Special Paper 284, 21-40.

Merschat, A.J., Hatcher Jr., R.D., 2007. The Cat Square terrane: Possible Siluro-Devonian remnant ocean basin in the Inner Piedmont, southern Appalachians, USA. In: Hatcher Jr., R.D., Carlson, M.P., McBride, J.H., Martínez Catalán, J.R. (Eds.), 4-D Framework of continental crustGeological Society of America Memoir 200, 553-565.

Murphy, J.B., Nance, R.D., 2002. Sm-Nd isotopic systematics as tectonic tracers: an example from West Avalonia in the Canadian Appalachians. Earth-Science Reviews 59, $77-100$.

Murphy, J.B., Keppie, J.D., Nance, R.D., Dostal, J., 2010. Comparative evolution of the Iapetus and Rheic Oceans: A North America perspective. Gondwana Research 17 $189-192$.

Nakamura, N., 1974. Determination of REE, Ba, Fe, $\mathrm{Mg}$, Na and $\mathrm{K}$ in carbonaceous and ordinary chondrites. Geochimica et Cosmochimica Acta 38, 757-775.

Nance, R.D., Gutiérrez-Alonso, G., Keppie, J.D., Linnemann, U., Murphy, J.B., Quesada, C. Strachan, R.A., Woodcock, N.H., 2010. Evolution of the Rheic Ocean. Gondwana Research 17, 194-222.

Potrel, A, Peucat, J.J, Fanning, C.M., 1998. Archean crustal evolution of the West African Craton: example of the Amsaga Area (Reguibat Rise). U-Pb and Sm-Nd evidence for crustal growth and recycling. Precambrian Research 90, 107-117.

Rodríguez Aller, J., 2005. Recristalización y deformación de litologías supracorticales sometidas a metamorfismo de alta presión (Complejo de Malpica-Tuy, NO del Macizo Ibérico). Nova Terra 29572 pp.

Rodríguez, J., Cosca, M.A., Gil Ibarguchi, J.L, Dallmeyer, R.D., 2003. Strain partitioning and preservation of ${ }^{4} \mathrm{Ar} /{ }^{39} \mathrm{Ar}$ ages during Variscan exhumation of a subducted crust (Malpica-Tui complex, NW Spain). Lithos 70, 111-139.

Rodríguez, J., Paquette,J.L., Gil Ibarguch i,J.L, 2007. U-Pb dating of Iower Ordovician alkaline magmatism in the Gondwana margin (Malpica-Tui Complex, Iberian
Massif): Latest continental events before oceanic spreading. In: Arenas, R., Martínez Catalán, J.R., Abati, J., Sánchez Martínez, S. (Eds.), The rootless Variscan suture of NW Iberia (Galicia Spain). Field trip guide and conference abstracts. International Geological Correlation Programme 497. The Rheic Ocean: Its origin, evolution and correlatives. Publicaciones del Instituto Geológico y Minero de España, pp. 163-164.

Rodríguez-Alonso, M.D., Peinado, M., López-Plaza, M., Franco, P., Carnicero, A, Gonzálo, J.C. 2004. Neoproterozoic-Cambrian synsedimentary magmatism in the Central Iberian Zone (Spain): geology, petrology and geodynamic significance. International Journal of Earth Sciences 93, 897-920.

Roser, B.P. Korsch, R.J. 1986. Determination of tectonic setting of sansdtone-mudstone suites using $\mathrm{SiO}_{2}$ content and $\mathrm{K}_{2} \mathrm{O} / \mathrm{Na}_{2} \mathrm{O}$ ratio. Journal of Geology 94, 635-650.

Roser, B.P. Korsch, R.J. 1988. Provenance signatures of sandstone-mudstone suites determined using discriminant function analysis of major-element data. Chemical Geology 67, 119-139.

Sánchez Martínez, S., Arenas, R., Díaz García, S., Martínez Catalán, J.R., Gómez Barreiro, J., Pearce,J., 2007. The Careón Ophiolite, NW Spain: supra-subduction zone setting for the youngest Rheic Ocean floor. Geology 35, 53-56.

Searle, M.P., 2007. Diagnostic features and processes in the construction and evolution of Oman, Zagros, Himalayan, Karakoram and Tibetan-type orogenic belts. In: Hatcher Jr., R.D., Carlson, M.P., McBride, J.H., Martínez Catalán, J.R. (Eds.), 4-D Framework of continental crustGeological Society of America Memoir 200, 1-7.

Stampfli, G.M., Borel, G.D., 2002. A plate tectonic model for the Paleozoic and Mesozoic constrained by dynamic plate boundaries and restored synthetic oceanic isochrons. Earth and Planetary Science Letters 196, 17-33.

Stern, R.J., 2002. Crustal evolution in the East African Orogen: a neodymium isotopic perspective. Journal of African Earth Sciences 34, 109-117.

Strelow, F.W.E., 1960. An ion exchange selectivity scale of cations based on equilibrium distributions coefficients. Analytical Chemistry 32, 1185-1188.

Taylor, S.R., McIennan, S.M., 1985. The Continental Crust: Its Composition and Evolution. Blackwell, London. $312 \mathrm{pp}$.

Thompson, R.N., 1982. Magmatism in the British Tertiary Volcanic Province. Scottish Journal of Geology 19, 49-107.

Ugidos,J.M., Valladares, M.I., Barba, P., Ellam, R.M., 2003. The Upper Neoproterozoic-Lower Cambrian of the Central Iberian Zone, Spain: Chemical and isotopic ( $\mathrm{Sm}-\mathrm{Nd}$ ) evidence that the sedimentary succesion reoords an inverted stratigraphy of its source. Geochimica et Cosmochimica Acta 67, 2615-2629.

Winchester, J.W., 1963. Rare earth chromatography using bis-(2-ethylhexyl) (orthophosphoric acid). Journal of Chromatography 10, 502-506.

Winchester, J.A., Max, M.D., 1989. Tectonic setting discrimination in clastic sequence: an example from the late proterozoic Erris Group, NW Ireland. Precambrian Research 45, 191-201. 